\title{
Dynamic control of a central pattern generator circuit: a computational model of the snail feeding network
}

\author{
Dimitris V. Vavoulis, ${ }^{1,2}$ Volko A. Straub, ${ }^{1, *}$ Ildikó Kemenes, ${ }^{1}$ György Kemenes, ${ }^{1}$ Jianfeng Feng, ${ }^{2, \dagger}$ and Paul R. \\ Benjamin $^{1}$ \\ ${ }^{1}$ Sussex Centre for Neuroscience, School of Life Sciences, University of Sussex, Falmer, Brighton, East Sussex, BN1 9QG, UK \\ ${ }^{2}$ Department of Informatics, University of Sussex, Brighton, UK
}

Keywords: dynamic control through inhibition, feeding motor systems, frequency control, Hodgkin-Huxley models, Lymnaea stagnalis

\begin{abstract}
Central pattern generators (CPGs) are networks underlying rhythmic motor behaviours and they are dynamically regulated by neuronal elements that are extrinsic or intrinsic to the rhythmogenic circuit. In the feeding system of the pond snail, Lymnaea stagnalis, the extrinsic slow oscillator (SO) interneuron controls the frequency of the feeding rhythm and the N3t (tonic) has a dual role; it is an intrinsic CPG interneuron, but it also suppresses CPG activity in the absence of food, acting as a decision-making element in the feeding circuit. The firing patterns of the SO and N3t neurons and their synaptic connections with the rest of the CPG are known, but how these regulate network function is not well understood. This was investigated by building a computer model of the feeding network based on a minimum number of cells (N1M, N2v and N3t) required to generate the three-phase motor rhythm together with the SO that was used to activate the system. The intrinsic properties of individual neurons were represented using twocompartment models containing currents of the Hodgkin-Huxley type. Manipulations of neuronal activity in the N3t and SO neurons in the model produced similar quantitative effects to food and electrical stimulation in the biological network indicating that the model is a useful tool for studying the dynamic properties of the feeding circuit. The model also predicted novel effects of electrical stimulation of two CPG interneurons (N1M and N2v). When tested experimentally, similar effects were found in the biological system providing further validation of our model.
\end{abstract}

\section{Introduction}

Research aimed at unravelling fundamental structural and functional principles of nervous system organization often focuses on central pattern generators (CPGs), i.e. networks of neurons that generate rhythmic motor behaviours, such as breathing, feeding or swimming. CPG networks are regulated dynamically by modulatory elements from within or outside the rhythmogenic circuit (Katz \& Frost, 1996; Marder \& Calabrese, 1996). This regulation may change the output patterns of a particular CPG or result in the complete reconfiguration of networks involving several different unit oscillatory circuits. Computational modelling of CPG networks has proved important in assessing the key parameters of CPG function (such as the ionic conductances required for the firing patterns of individual component neurons) and in evaluating the role of specific types of synaptic connectivity in producing network output (Marder \& Selverston, 1992; Marder \& Abbott, 1995; Abbott \& Marder, 1998; Destexhe \& Marder, 2004). Computer simulations can also provide a useful tool for studying the dynamic properties of CPG circuits, particularly if

Correspondence: Dr Dimitris V. Vavoulis, as above.

E-mail: D.Vavoulis@sussex.ac.uk

* Present address: Department of Cell Physiology and Pharmacology, University of Leicester, Leicester, UK

${ }^{\dagger}$ Present address: Centre for Scientific Computing, Warwick University, Coventry, UK

Received 15 January 2007, revised 26 February 2007, accepted 6 March 2007 modelling studies can be tightly coupled to experimental data (Marder \& Abbott, 1995).

The present study aimed to model the network properties of two types of neurons that control the CPG-driven motor pattern underlying feeding in the pond snail, Lymnaea (Fig. 1). One type of cell, the slow oscillator (SO), is extrinsic to the CPG network and controls the frequency of the feeding oscillator via known synaptic connectivity with CPG interneurons (Rose \& Benjamin, 1981; Kemenes et al., 2001). The other type of cell, the N3 tonic (N3t), is intrinsic to the CPG network but also plays a role in suppressing CPG activity in the absence of food (Staras et al., 2003). Feeding in Lymnaea is an episodic motor behaviour with bouts of feeding interspersed with periods of quiescence. Quiescence arises from active inhibition of the CPG by N3t, which fires tonically to inhibit another CPG interneuron, the N1 medial (N1M). In the presence of food (sucrose), tonic activity in N3t is suppressed and the cell switches function to fire phasically as part of the food-evoked rhythmic activity. Thus, N3t plays a dual role as a decision-making neuron determining the occurrence of episodic feeding behaviour (Calabrese, 2003), as well as functioning as a CPG interneuron (Elliott \& Benjamin, 1985a).

Understanding the dynamic control functions of the SO and N3t cells required building a basic model of the Lymnaea feeding CPG. We modelled the intrinsic properties of SO, N3t and two other CPG interneuron types, N1M and N2v (N2 ventral) using two-compartment models based on Hodgkin-Huxley equations for the representation of ionic currents (Hodgkin \& Huxley, 1952). We then organized these 


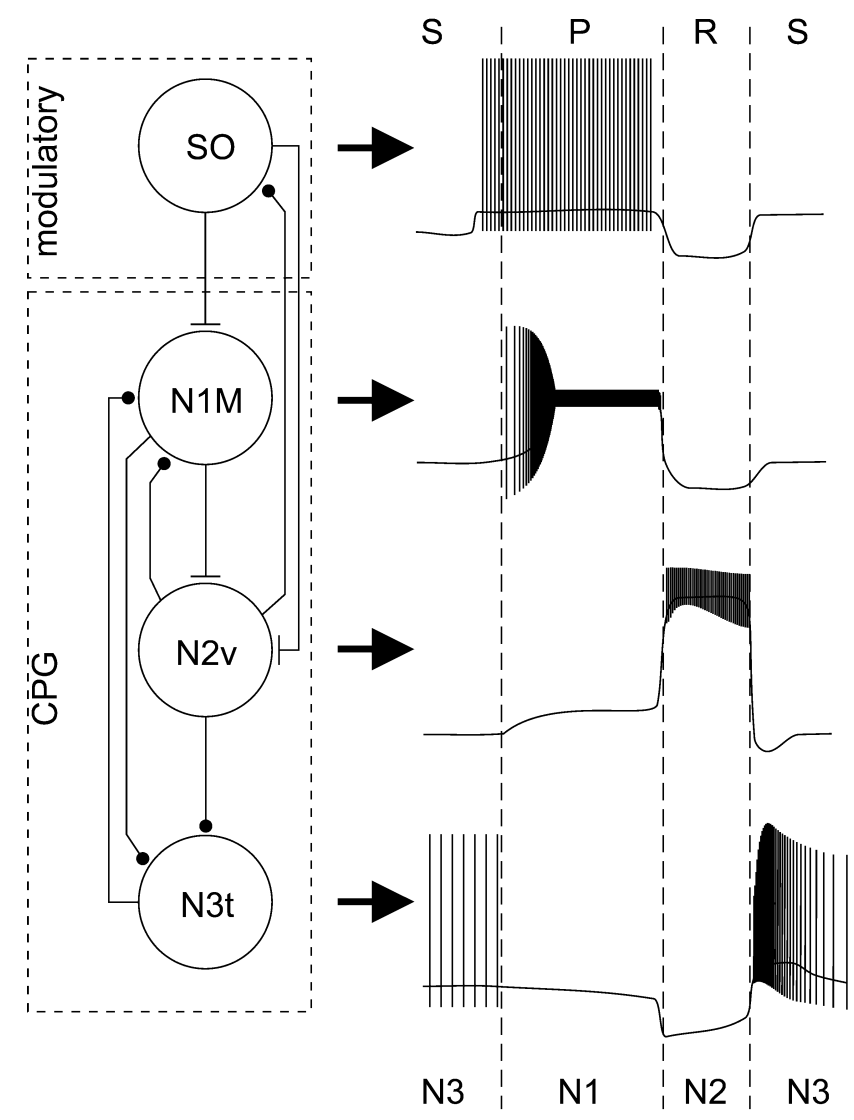

FIG. 1. Summary of the firing patterns and synaptic connectivity of the Lymnaea feeding CPG (Brierley et al., 1997b; Straub et al., 2002). Feeding is achieved by the repetition of a basic triphasic pattern of electrical activity in feeding CPG interneurons, the N1, N2 and N3 cells. The most important neurons from each group ( $\mathrm{N} 1$ medial or $\mathrm{N} 1 \mathrm{M}, \mathrm{N} 2$ ventral or $\mathrm{N} 2 \mathrm{v}, \mathrm{N} 3$ tonic or $\mathrm{N} 3 \mathrm{t}$ ) and the synapses between them are illustrated (left; bars indicate excitation, circles indicate inhibition). The slow oscillator (SO) is a modulatory interneuron that controls the frequency of the feeding rhythm. A schematic diagram of the rhythmic electrical activity of the CPG is illustrated (right), showing that the feeding cycle is divided into three phases, protraction (P), rasp (R) and swallow (S), each corresponding to phases of the behavioural feeding rhythm. The pattern has been generated by constant current injection into SO (SO-driven rhythm).

artificial neurons into a minimal network configuration, reflecting the known topology of the biological circuit and capable of producing the three phase feeding pattern. The intrinsic and synaptic properties of the $\mathrm{N}$ cells and SO have been extensively studied in the intact ganglion and in cell culture (e.g. Elliott \& Benjamin, 1985a, b; Straub et al., 2002) by paired recordings and current clamp techniques and these data guided the modelling study.

Using this model network, we found that N3t was capable of mediating regulatory effects upon the basic rhythmogenic properties of the CPG model in agreement with independently obtained experimental data (Staras et al., 2003), providing support for the conclusion that $\mathrm{N} 3 \mathrm{t}$ is a hybrid pattern-generating/regulatory interneuron. We also showed that high-frequency oscillations, driven by SO, are achieved by the model network via specific changes in the duration of two phases of the feeding rhythm, but not the third, mimicking independent experimental results published earlier (Elliott \& Andrew, 1991). Further validation of the model was obtained by correctly predicting the ability of two of the CPG neurons (N1M and N2v) to influence the frequency of the feeding rhythm when we carried out subsequent electrophysiological experiments in the natural system.

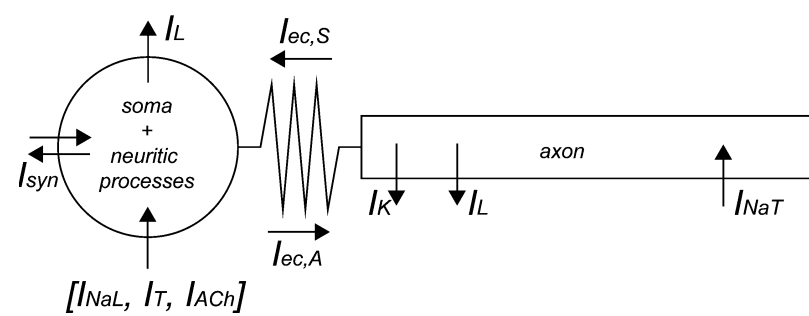

FIG. 2. General structure of the neuronal model. Each neuron in the network was represented by a two-compartment, conductance-based model, which assumes a spatial separation between fast (action potentials) and slow (postinhibitory rebound, plateau potentials) processes. The soma + neuritic processes compartment represents the cell body and the site of synaptic connections with other neurons. Located in this compartment are slow conductances $\left(\left[I_{A C h}\right.\right.$, $\left.I_{N a L}, I_{T} J\right)$, which are responsible for low-amplitude, slow-developing changes of the membrane potential, such as plateau potentials or post-inhibitory rebound. The brackets indicate that these slow currents may be absent in a cell like SO, where the somatic compartment is completely passive. $I_{\text {syn }}$ is the synaptic current induced by presynaptic activity. The compartment labelled axon represents the more distal, spike-initiating regions of the neuron and it contains the appropriate conductances $\left(I_{L}, I_{N a T}, I_{K}\right)$ that are responsible for spike generation. Action potentials recorded at the soma are assumed to be electrotonic remnants of events initiated in this compartment. Both compartments contain additional currents $\left(I_{e c, S}, I_{e c, \mathrm{~A}}\right)$ due to the electrotonic coupling between them. A justification of the two-compartment approach is given in the main text. The direction of the arrow(s) indicates that a current is either inward or outward. Adjacent arrows in the opposite direction indicate that a current has a combination of inward and outward components.

\section{Materials and methods Overview}

The network we model here contains the most important neurons from each of the three groups of CPG interneurons (i.e. N1M, N2v and N3t) and the modulatory SO interneuron. The individual neurons of the CPG were represented by two-compartment models (Fig. 2), which contain currents of the type initially introduced by Hodgkin \& Huxley (1952). The first compartment, labelled 'soma + neuritic processes', represents the soma and proximal neuritic branching and it may be either passive (containing only a leakage current, $I_{L}$, as in the case of SO) or it may contain additional conductances $\left(I_{A C h}, I_{N a L}\right.$ or $\left.I_{T}\right)$, which underlie slowly developing, long-lasting changes of the membrane potential (Elliott \& Benjamin, 1985a), such as plateau potentials (N1M and N2v) or post-inhibitory rebound (N3t). In addition, it is in this compartment where synaptic contacts with other CPG interneurons are located (Elliott \& Benjamin, 1985a). Inward and outward currents $\left(I_{s y n}\right)$ underlie synaptic events in the model. The second compartment, labelled 'axon', represents the distal, spike-initiating parts of the neuron and, apart from a leakage conductance $I_{L}$, it contains fast currents $\left(I_{N a T}, I_{K}\right)$, which are responsible for the generation of action potentials. Both compartments contain additional currents $\left(I_{e c, S}, I_{e c, A}\right)$, which model the electrotonic coupling between them. Therefore, all neurons in the model CPG have the same structure, being different only in the properties of the somatic compartment and the strength of the electrotonic coupling between this compartment and the axon.

A two-compartment model is the simplest possible way to represent the spatial separation between different functions within a single neuron and, in our case there are several reasons that justify this approach. First of all, there is direct evidence from studies in culture (Straub, 1999; Straub et al., 2002) that different parts of the CPG interneurons express different electrical properties. For example, the inability of isolated $\mathrm{N} 2 \mathrm{v}$ somata to generate plateau potentials suggests that the relevant conductances are located in distal parts of the cell that are lost during the isolation procedure. Second, the action potentials 
recorded at the soma (and, indeed, at every part of the neuron that is confined within the buccal ganglia) are small in size and, typically, they barely overshoot $0 \mathrm{mv}$ (e.g. Straub et al., 2002; Staras et al., 2003), which suggests that they are electrotonic remnants of spikes generated at distal parts of the neuron. Finally, the recorded electrical activity of the CPG interneurons often appears to consist of an underlying depolarization, upon which a series of full or truncated action potentials are superimposed, such as the N1M and N2v plateau potentials or the N3t post-inhibitory rebound (e.g. Yeoman et al., 1995; Brierley et al., 1997a, b; Straub et al., 2002; Staras et al., 2003). This characteristic activity can be reproduced if we assume that the two elements that characterize the recording (i.e. underlying depolarization and superimposed spikes) are generated at spatially distinct, but electrotonically connected regions of the cell. This spatial distribution of different cellular properties minimizes the interference between fast and slow conductances, thus leading to more stable behaviours (Abbott \& Marder, 1998). Functional variations between different parts of an invertebrate (unipolar) neuron are known to occur in a number of cases justifying a multicompartment approach (see Marder \& Selverston, 1992; Abbott \& Marder, 1998; for a general discussion; Booth et al., 1997; Golowasch et al., 1999; Susswein et al., 2002; Soto-Trevino et al., 2005; for specific examples in both vertebrate and invertebrate systems).

\section{Mathematical framework}

The equation for the conservation of current holds for both the somatic and the axonal compartments. In the case of the somatic compartment, it takes the following form (Table 1):

$$
\tau_{S} \frac{d V_{S}}{d t}=i_{i n j}-i_{L, S}-i_{X}-i_{e c, S}-i_{s y n}
$$

where $V_{S}$ is the membrane potential of the somatic compartment (in $\mathrm{mV}$ ) and $\tau_{s}$ is the passive time constant of the membrane (in ms), given by the ratio of the somatic membrane capacitance (in $\mathrm{nF}$ ) and leakage conductance (in $\mu$ S), i.e. $\tau_{S}=C_{S} / \bar{g}_{L, S}$.

$i_{i n j}$ is the product of the externally applied current (e.g. via an electrode, in $\mathrm{nA}$ ) and the passive input resistance (in $\mathrm{M} \Omega$ ) of the somatic compartment, i.e. $i_{i n j}=I_{i n j} \times R_{S}$ (where $\left.R_{S}=1 / \bar{g}_{L, S}\right)$ and it is measured in $\mathrm{mV}$.

The quantity $i_{L, S}=\left(V_{S}-E_{L}\right)$ is the product of the leakage current $I_{L, S}=\bar{g}_{L, S} \times\left(V_{S}-E_{L}\right)$ and the somatic input resistance $R_{S}$, where $E_{L}$ is the leakage reversal potential.

$i_{x}$ represents any of the slow, located-at-the-soma conductances $\left(I_{A C h}, I_{N a L}\right.$ or $I_{T}$ in Fig. 2), which are responsible for slowly developing, long-lasting properties such as plateau potentials and postinhibitory rebound, and it takes the following general form:

$$
i_{X}=\bar{\gamma}_{X} p_{X}{ }^{k_{X}} q_{X}{ }^{l_{X}}\left(V_{S}-E_{X}\right), \quad X=A C h, N a L, T
$$

where $p_{x}$ and $q_{x}$ are dynamic activation and inactivation variables, $k_{x}$ and $l_{x}$ are integers, which take values from the set $\{0,1,2,3,4\}$ and $E_{X}$ is the reversal potential of the current. $\bar{\gamma}_{X}$ is the dimensionless product $\bar{g}_{X} \times R_{S}$, where $\bar{g}_{X}$ is the maximal conductance of the current (in $\mu \mathrm{S}$ ). The dynamic variables $p_{x}$ and $q_{x}$ follow first-order relaxation kinetics:

$$
\frac{d u_{i}}{d t}=\frac{u_{\infty, i}-u_{i}}{\tau_{u, i}}, \quad u=p, q
$$

Where the steady-states $u_{\infty, i}$ and the relaxation time constants $\tau_{u, i}$ are, in general, voltage-dependent and modelled using sigmoid or Gaussian functions (Table 1).

$i_{e c, S}$ represents the current entering the soma due to its electrotonic coupling with the axonal compartment and it is equal to $\bar{\gamma}_{e c, S} \times\left(V_{S}-V_{A}\right) . \quad V_{A}$ is the membrane potential of the axonal compartment and $\bar{\gamma}_{e c, S}$ is the product of the electrotonic coupling conductance $\bar{g}_{e c, S}$ in the axon $\rightarrow$ soma direction times the passive somatic input resistance $R_{S}$.

Finally, $i_{\text {syn }}$ represents the total synaptic current received by the neuron and it is given by the following expression (Table 2):

$$
i_{s y n}=\sum_{j} \bar{\gamma}_{s y n, j} s_{j}\left(V_{S}-E_{s y n, j}\right)
$$

where $E_{s y n, j}$ is the reversal potential of the $j^{\text {th }}$ synaptic current (equal to

\begin{tabular}{|c|c|c|c|c|}
\hline Neuron & Intrinsic currents & Dynamic variables & Steady states & Relaxation time constants (ms) \\
\hline N1M & $\begin{array}{l}i_{A C h}=200 \times p^{3} \times\left(V_{S}+30\right) \\
i_{L, S}=\left(V_{S}+67\right) \\
i_{e c, S}=8 \times\left(V_{S}-V_{A}\right)\end{array}$ & $d p / d t=\left(p_{\infty}-p\right) / \tau_{p}$ & $p_{\infty}=1 /\left(1+\exp \left(\left(-38.8-V_{S}\right) / 10\right)\right)$ & $\tau_{p}=250$ \\
\hline $\mathrm{N} 2 \mathrm{v}$ & $\begin{array}{l}i_{N a L}=2 \times p^{3} \times q \times\left(V_{S}-55\right) \\
i_{L, S}=\left(V_{S}+67\right) \\
i_{e c, S}=0.55 \times\left(V_{S}-V_{A}\right)\end{array}$ & $\begin{array}{l}d p / d t=\left(p_{\infty}-p\right) / \tau_{p} \\
d q / d t=\left(q_{\infty}-q\right) / \tau_{q}\end{array}$ & $\begin{aligned} p_{\infty} & =1 /\left(1+\exp \left(\left(-51-V_{S}\right) / 10.3\right)\right) \\
q_{\infty} & =1 /\left(1+\exp \left(\left(-45-V_{S}\right) /-3\right)\right)\end{aligned}$ & $\begin{array}{l}\tau_{p}=28.3+44.1 \times \exp \left(-\left(\left(-11.8-V_{A}\right) / 26.6\right)^{2}\right) \\
\tau_{q}=187.6+637.7 \times \exp \left(-\left(\left(-9.5-V_{A}\right) / 23.3\right)^{2}\right)\end{array}$ \\
\hline $\mathrm{N} 3 \mathrm{t}^{\ddagger}$ & $\begin{array}{l}i_{T}=3.27 \times p^{3} \times q \times\left(V_{S}-80\right) \\
i_{L, S}=\left(V_{S}+67\right) \\
i_{e c, S}=8 \times\left(V_{S}-V_{A}\right)\end{array}$ & $\begin{array}{l}d p / d t=\left(p_{\infty}-p\right) / \tau_{p} \\
d q / d t=\left(q_{\infty}-q\right) / \tau_{q}\end{array}$ & $\begin{aligned} p_{\infty} & =1 /\left(1+\exp \left(\left(-61.6-V_{S}\right) / 5.6\right)\right) \\
q_{\infty} & =1 /\left(1+\exp \left(\left(-73.2-V_{S}\right) /-5.1\right)\right)\end{aligned}$ & $\begin{array}{l}\tau_{p}=4 \\
\tau_{q}=400\end{array}$ \\
\hline SO & $\begin{array}{l}i_{L, S}=\left(V_{S}+67\right) \\
i_{e c, S}=8 \times\left(V_{S}-V_{A}\right)\end{array}$ & & & \\
\hline
\end{tabular}
$0 \mathrm{mV}$ in the case of an excitatory synapse, $-90 \mathrm{mV}$ in the case of an inhibitory one) and, similarly to the other currents, $\bar{\gamma}_{s y n, j}$ is the product

TABLE 1. Summary of the intrinsic properties and parameter values of the somatic compartment*

${ }^{\ddagger}$ The set of simulations in Fig. $6 \mathrm{~B}$ required the absence of any significant post-inhibitory rebound response (see main text for justification). Thus, in this case only, $i_{T}=0$. ${ }^{*}$ With $\tau_{\mathrm{m}}=10 \mathrm{~ms}$ throughout. 
TABLE 2. Summary of the synaptic properties and parameters values of the model

\begin{tabular}{|c|c|c|c|c|c|}
\hline Postsynaptic & Presynaptic & Polarity & Maximal synaptic conductance $\left(\bar{\gamma}_{\text {syn }}\right)$ & Reversal potential (mV) & Activation time constant (ms) \\
\hline $\mathrm{SO}$ & $\mathrm{N} 2 \mathrm{v}$ & Inhibitory & 8 & -90 & 50 \\
\hline \multirow[t]{3}{*}{ N1M } & $\mathrm{SO}$ & Excitatory & 4 & 0 & 200 \\
\hline & $\mathrm{N} 2 \mathrm{v}$ & Inhibitory & 50 & -90 & 50 \\
\hline & N3t & Inhibitory & 8 & -90 & 50 \\
\hline \multirow[t]{2}{*}{$\mathrm{N} 2 \mathrm{v}$} & SO & Excitatory & 1.0 & 0 & 200 \\
\hline & N1M & Excitatory & 0.077 & 0 & 200 \\
\hline \multirow[t]{2}{*}{ N3t } & N1M & Inhibitory & 0.5 & -90 & 50 \\
\hline & $\mathrm{N} 2 \mathrm{v}$ & Inhibitory & 2 & -90 & 50 \\
\hline
\end{tabular}

of the $j^{\text {th }}$ maximal synaptic conductance $\bar{g}_{s y n, j}$ and the passive input resistance of the soma $R_{S}$. The dynamic variable $s_{j}$ represents the activation of the $j^{\text {th }}$ synapse and it is modelled as a second-order, differential-equation formulation of the $\alpha$-function (Wilson, 1999):

$$
\begin{gathered}
\frac{d r_{j}}{d t}=\frac{r_{\infty, j}-r_{j}}{\tau_{s y n, j}} \\
\frac{d s_{j}}{d t}=\frac{r_{j}-s_{j}}{\tau_{s y n, j}}
\end{gathered}
$$

where $\tau_{s y n, j}$ is the characteristic activation time constant of the $j^{\text {th }}$ synaptic current. Typically, in the case of spike-mediated synaptic transmission, the steady-state value $r_{\infty, j}$ takes the value 1 during a presynaptic spike, otherwise it takes the value 0 . In our case, it was assumed that synaptic transmission takes place in a graded, non-spikemediated manner. This assumption is justified by the fact that (i) the low-amplitude membrane potentials that are recorded from the presynaptic compartments (soma and proximal neuritic processes) are sufficient to induce significant responses to postsynaptic cells, even in the absence of full action potentials (e.g. Elliot \& Benjamin, 1985a; Staras et al., 1998; Straub, 1999) and (ii) non-spike mediated synaptic transmission has been directly demonstrated in cell culture (Straub, 1999). In accordance with these experimental results, $r_{\infty, j}$ was assumed to be a sigmoid function of the presynaptic potential $V_{\text {pre }}$ :

$$
r_{\infty, j}=\frac{1}{1+e^{\left(-40-V_{\text {pre }}\right) / 2.5}}
$$

which allows for the activation of the synapse even by relatively small depolarizations of the presynaptic membrane and in a graded, rather than an all-or-none, fashion.

The axonal compartment contains the necessary conductances for the generation of action potentials and, similarly to the soma, the current conservation equation takes the following form (Table 3):

$$
\tau_{A} \frac{d V_{A}}{d t}=-i_{L, A}-i_{N a T}-i_{K}-i_{e c, A}
$$

where $\tau_{A}, V_{A}$ and $i_{L, A}$ are defined similarly to the corresponding quantities of the somatic compartment. $i_{N a T}$ and $i_{k}$ represent a fast, inactivating sodium current and a delayed rectifier potassium current, respectively. $i_{N a T}$ is equal to $\bar{\gamma}_{N a T} \times m_{\infty}{ }^{3} \times h \times\left(V_{A}-E_{N a}\right)$, where $E_{N a}$ is the sodium reversal potential and $\bar{\gamma}_{N a T}$ is the product of the maximum sodium conductance $\bar{g}_{N a T}$ times the passive input resistance of the axon, $R_{A}=1 / \bar{g}_{L, A}$ (here, $\bar{g}_{L, A}$ is the leakage conductance of the axonal compartment). Similarly, $i_{k}$ is given by the expression $\bar{\gamma}_{K} \times n^{4} \times\left(V_{A}-E_{K}\right)$, where $\bar{\gamma}_{K}$ and $E_{K}$ are defined as for the sodium current. The dynamic variables $h$ and $n$ follow first-order relaxation kinetics:

$$
\frac{d u}{d t}=\frac{u_{\infty}-u}{\tau_{u}}, \quad u=h, n
$$

The steady-state quantities $m_{\infty}, u_{\infty}$ and the relaxation time constants $\tau_{u}$ are dependent on the axonal membrane potential $V_{A}$ and modelled using sigmoid or Gaussian functions.

The form and parameters of $i_{L, A}, i_{N a T}$ and $i_{k}$ were chosen such that the axonal compartment exhibits Class I excitability, according to Hodgkin's classification (Hodgkin, 1948), and they are modified from the Wang \& Buzsaki (1996) spiking model. This decision was based on the observation that the firing rate of the neurons that are being modelled often demonstrates smooth transitions, e.g. during the initial stages of the N1M plateau potential or the gradual termination of the N3t post-inhibitory rebound (see Fig. 3), which suggests the presence of a Class I firing mechanism.

Finally, the current due to the electrotonic coupling with the soma, $i_{e c, A}$, is equal to $\bar{\gamma}_{e c, A} \times\left(V_{A}-V_{S}\right)$, where $\bar{\gamma}_{e c, A}$ is the product of the electrotonic coupling conductance $\bar{g}_{e c, A}$ in the soma $\rightarrow$ axon direction and the axonal passive input resistance $R_{A}$.

In the model, current is measured in $\mathrm{nA}$, capacitance in $\mathrm{nF}$, conductance in $\mu \mathrm{S}$, time in $\mathrm{ms}$ and the membrane potential in $\mathrm{mV}$. The quantity $i$ is always the product of a current $I$ (in $\mathrm{nA}$ ) times a passive input resistance $R$ (in $\mathrm{M} \Omega$ ) and it is also measured in $\mathrm{mV}$. Finally, the quantity $\bar{\gamma}$ is given without units.

\section{Simulations}

\begin{tabular}{|c|c|c|c|c|}
\hline Neuron & Intrinsic currents & Dynamic variables & Steady states & Relaxation time constants (ms) \\
\hline $\begin{array}{l}\mathrm{N} 1 \mathrm{M} \\
\mathrm{N} 3 \mathrm{t} \\
\mathrm{SO} \\
\mathrm{N} 2 \mathrm{v}^{\ddagger}\end{array}$ & $\begin{array}{l}i_{N a T}=350 \times m_{\infty}^{3} \times h \times\left(V_{A}-55\right) \\
i_{K}=90 \times n^{4} \times\left(V_{A}+90\right) \\
i_{L, A}=\left(V_{A}+67\right) \\
i_{e c, A}=8 \times\left(V_{A}-V_{S}\right)\end{array}$ & $\begin{aligned} d h / d t & =\left(h_{\infty}-h\right) / \tau_{h} \\
d n / d t & =\left(n_{\infty}-n\right) / \tau_{n}\end{aligned}$ & $\begin{array}{l}m_{\infty}=1 / 1+\exp \left(\left(-34.6-V_{A} / 9.6\right)\right) \\
h_{\infty}=1 /\left(1+\exp \left(\left(-55.2-V_{A}\right) /-7.1\right)\right) \\
n_{\infty}=1 /\left(1+\exp \left(\left(-30-V_{A}\right) / 17.4\right)\right)\end{array}$ & $\begin{array}{l}\tau_{h}=1.1+7.2 \times \exp \left(-\left(\left(-61.3-V_{A}\right) / 22.7\right)^{2}\right) \\
\tau_{n}=1.1+4.6 \times \exp \left(-\left(\left(-61-V_{A}\right) / 54.3\right)^{2}\right)\end{array}$ \\
\hline
\end{tabular}

The CPG model took the form of a 38-dimensional system of ordinary differential equations (ODEs), which were encoded and solved using custom code written in the programming language ANSI $\mathrm{C}$ and the

TABLE 3. Summary of the intrinsic properties and parameter values of the axonal compartment*

*In the case of $\mathrm{N} 2 \mathrm{v}$ only, $i_{e c, A}=0.06 \times\left(V_{A}-V_{S}\right) .{ }^{*}$ With $\tau_{\mathrm{m}}=10 \mathrm{~ms}$ throughout. 
A N1M

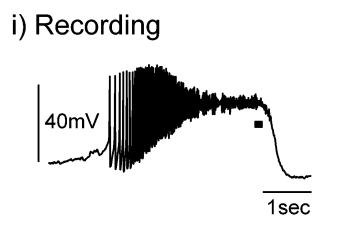

ii) Simulation
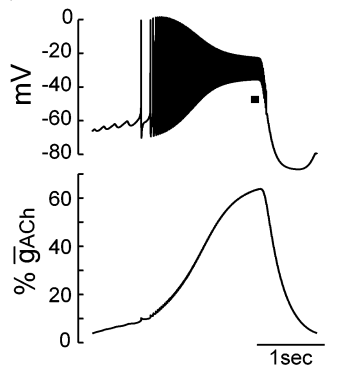

C N3t

i) Recording

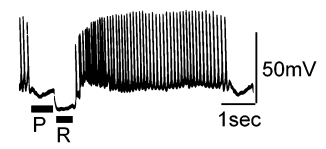

ii) Simulation

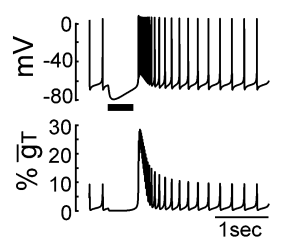

iii) The role of EAch

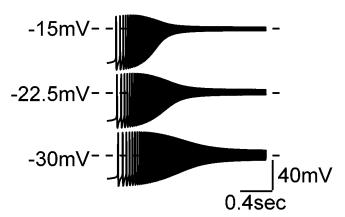

iv) F-I relationship

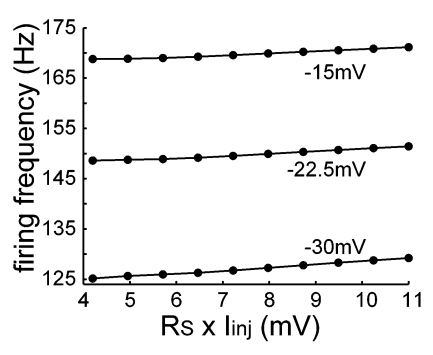

iii) Response to inhibitory pulses

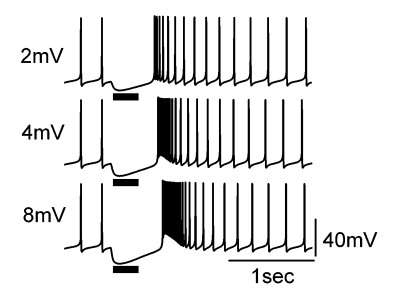

B N2v

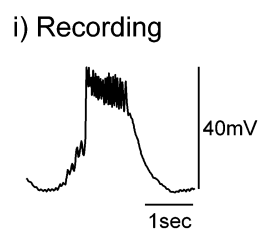

ii) Simulation

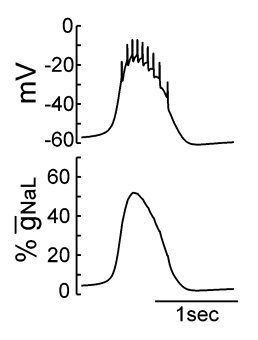

iii) Response to injected current

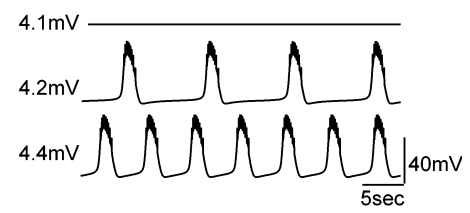

iv) F-I relationship

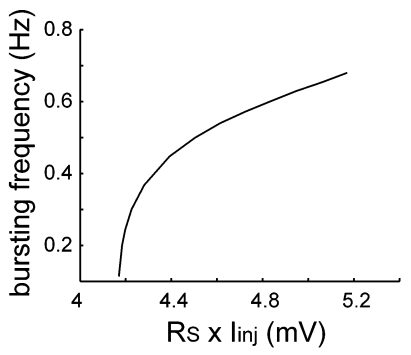

D so

i) Recording

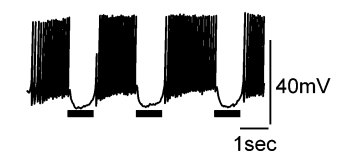

ii) Simulation

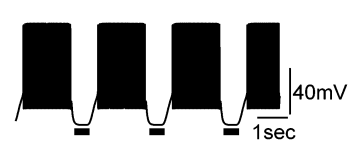

iii) F-I relationship

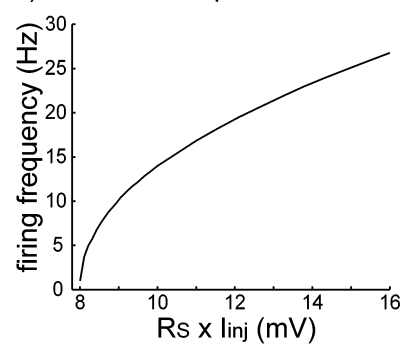

FIG. 3. Neuronal models for the N1M (A), N2v (B), N3t (C) and SO (D) interneurons. In panels A, B and C, (i) shows an intracellular recording of the corresponding neuron and (ii) illustrates the characteristic (for each neuron) activity at the soma (top), along with a key underlying ionic conductance (bottom). The rest of the figure presents the characteristic electrical properties of each model neuron; (A) the dependence of the N1M plateau on the reversal potential of the plateau-sustaining (depolarizing) current $i_{A C h}$ (iii, $E_{A C h},-15 \mathrm{mV},-22.5 \mathrm{mV}$ and $-30 \mathrm{mV}$ ), and the relative insensitivity of the $\mathrm{N} 1 \mathrm{M}$ mean firing frequency (calculated over an interval of $5 \mathrm{~s}$ after neuronal activity commences) to external current injection, again at three different levels of $E_{A C h}$ (iv, $E_{A C h},-15 \mathrm{mV}$, $-22.5 \mathrm{mV}$ and $-30 \mathrm{mV}$ ); (B) the ability of the $\mathrm{N} 2 \mathrm{v}$ to oscillate at very low frequencies when minimally stimulated (iii, $i_{\text {inj; }}, 4.1 \mathrm{mV}, 4.2 \mathrm{mV}$ and $4.4 \mathrm{mV}$ ) and its current-frequency response (iv) and (C) the dependence of the N3t post-inhibitory rebound property on the strength of brief hyperpolarizing stimuli (iii, $i_{\text {inj }}$, $2 \mathrm{mV}$, $4 \mathrm{mV}$ and $8 \mathrm{mV}$ ). (D) An SO intracellular recording (i), the somatic activity of the SO model, interrupted by brief inhibitory pulses (ii) and its current-frequency (F-I) response (iii). For more details, see main text.

GNU Scientific Library (Galassi et al., 2005; http://www.gnu.org/ software/gsl/). All simulations were realized on a Pentium IV with $512 \mathrm{MB}$ of memory and the Linux operating system. The ODEs were solved using a Runge-Kutta-Fehlberg $(4,5)$ algorithm with adaptive step size and an absolute error tolerance of $10^{-6}$.

The parameters for the individual neuronal models were selected following specific physiological criteria (e.g. Class I excitability), in order to replicate the recorded behaviour of the neurons under current-clamp. Then, we assembled these models (keeping their parameters fixed) into networks of increasing complexity and we adjusted the strengths of the synaptic connections between the neurons based on electrophysiological data. Paired recordings between the CPG interneurons (e.g. Elliott \& Benjamin, 1985a) formed the basis for adjusting the characteristics (i.e. conductance, activation time constant) of each synapse. Overall, we had sufficient data to give reasonable values to the various parameters of the model.

\section{Sensitivity analysis}

The effect of key parameters on the behaviour of the individual neuronal models was investigated (e.g. Fig. 3A, iii and iv, for the N1M or Fig. 3B, iii and iv for the N2v), as well as the sensitivity of the complete network to current injection into any of its component neurons (e.g. Fig. 7B). The effect of modifying the $\mathrm{SO} \rightarrow \mathrm{N} 2 \mathrm{v}$ excitatory conductance on the response of the network to injected current was also systematically examined (Fig. 7C).

\section{Electrophysiology}

Standard intracellular recording techniques were used to stimulate and record feeding interneurons located in the buccal ganglia of laboratory-bred Lymnaea (e.g. Kemenes et al., 2001; Straub et al., 2002). CPG interneurons were identified on the basis of position and firing pattern as described in earlier papers (Benjamin \& Rose, 1979; Elliott \& Benjamin, 1985a, b; Brierley et al., 1997b). 


\section{Results}

\section{Modelling the intrinsic properties of the feeding interneurons}

N1M plateauing neurons

The NIM neurons fire during the first, protraction or $\mathrm{P}$ phase of the feeding cycle (Fig. 1). The characteristic pattern of N1M plateauing activity (Fig. 3, Ai) begins with a train of full-sized spikes, which gradually increase in frequency and decrease in amplitude. Progressively, the membrane potential reaches a sustained, depolarized state of approximately $-30 \mathrm{mV}$, upon which truncated action potentials are superimposed. In the intact nervous system, the plateau is terminated by inhibitory synaptic feedback from the N2 interneurons (Fig. 1). Cultured N1M cells retain their ability to generate plateau potentials indicating that this is an intrinsic property of these neurons (Straub et al., 2002). Moreover, it has been demonstrated (Straub, 1999) that the N1M plateau is both voltage- and ACh-dependent and reverses polarity at approximately $-30 \mathrm{mV}$.

In the model, it was assumed that the activation of a strong somatic inward current, $i_{A C h}$, is responsible for the generation of the plateau (in this neuron, $i_{X}=i_{A C h}$; Straub, 1999). No spike-generating conductances exist in the soma and the action potentials recorded here are back-propagated from the axonal compartment. This is the case for all the neurons modelled in the present study. The initial transitory phase of the plateau, which is marked by a progressive change in the frequency and size of the action potentials, reflects the slow (time constant, $250 \mathrm{~ms}$ ) activation of $i_{A C h}$ (Fig. 3, Aii). As a larger proportion of the current becomes activated and the membrane becomes depolarized, more current flows into the axonal compartment, and this explains the gradual increase in spike frequency (Fig. 3, Aii). An important element of this model is that $i_{A C h}$ has a reversal potential of approximately $-30 \mathrm{mV}$, i.e. the same value as the membrane potential after the plateau has reached its steady state. As the magnitude of the depolarizing current increases, it progressively overshadows all the other currents (saturation effect) and the membrane potential moves towards a new equilibrium point $(-30 \mathrm{mV})$, the reversal potential of $i_{A C h}$ As a result of this saturation effect, the size of the soma-recorded action potentials progressively decreases until the point where only a small electrotonic spike remnant remains, distributed around the equilibrium potential of the depolarizing current. As shown in Fig. 3, Aiii, the reversal potential of $i_{A C h}$ $\left(E_{A C h}\right)$ determines the value of the membrane potential that the plateau finally reaches.

If inhibitory stimuli drive the membrane potential to sufficiently negative values (e.g. more negative than $-60 \mathrm{mV}$ ), $i_{A C h}$ deactivates and the plateau is terminated.

In Fig. 3A, iv, we illustrate the mean firing frequency of the N1M (calculated over a time interval of $5 \mathrm{~s}$ after neuronal activity commences) as a function of injected current for three different values of $E_{A C h}(-15 \mathrm{mV},-22.5 \mathrm{mV}$ and $-30 \mathrm{mV})$. In all three cases, the mean firing frequency remains relatively constant (compare to SO, Fig. 3D, iii) with increasing current. This interesting property limits the ability of injected current to significantly affect the frequency of N1M-driven feeding rhythms (see below).

\section{$N 2 v$ plateauing neurons}

The $\mathrm{N} 2 \mathrm{v}$ neurons show plateau activity during the second, rasp or R phase of the feeding rhythm (Fig. 1). Compared to N1M, the N2v firing pattern is more conventional (Fig. 3B, i) and resembles other well-known plateauing CPG neurons, such as the anterior burster or pyloric dilator of the stomatogastric ganglion (e.g. Abbott \& Marder, 1998). The $\mathrm{N} 2 \mathrm{v}$ plateau consists of a rapid, $\sim 40 \mathrm{mV}$ membrane depolarization, upon which truncated action potential are superim- posed. The plateau terminates spontaneously, approximately $1 \mathrm{~s}$ after its initiation. In the intact nervous system, N2v cells are capable of producing plateaus, if artificially stimulated, even when synaptic input from the rest of the network has been blocked (Brierley et al., 1997a). This is a strong indication that the plateau-generating ability of the $\mathrm{N} 2 \mathrm{v}$ is intrinsic to these neurons. Furthermore, the plateau was shown to persist in zero $\mathrm{Ca}^{2+}$ saline (Brierley et al., 1997b).

In our model, we have assumed that a slowly activating, slowly inactivating sodium conductance $i_{N a L}$ is responsible for the generation of this characteristic behaviour $\left(i_{X}=i_{N a L}\right)$. A persistent sodium current has been found in the feeding CPG of Lymnaea (Staras et al., 2002) and it has been demonstrated sufficiently strong to support up to $\sim 40 \mathrm{mv}$ depolarizations of the membrane potential. The slow activation of $i_{N a L}$ is responsible for the depolarising phase of the plateau in the model, while the delayed inactivation of this current at more depolarized levels repolarises the membrane after approximately $1 \mathrm{~s}$ (Fig. 3B, ii). Both the activation and inactivation time constants of $i_{\text {NaL }}$ were modelled using Gaussian functions (Table 1). The parameter values we have chosen in the model reproduced the plateau shape of the biological neuron (see Fig. 3B, i) and allowed for the rapid recovery from the hyperpolarized state following the termination of the plateau, in agreement with the experimental recordings (Brierley et al., 1997a, b).

While the somatic compartment remains depolarized during the plateau, the axonal compartment is stimulated by current entering from the soma. The spikes generated in this way are electrotonically transmitted back to the somatic compartment and recorded as attenuated action potentials superimposed on the plateau depolarization.

As shown in Fig. 3B, iii, the model is capable of generating plateau potentials at regular intervals when injected with sufficiently strong constant depolarizing current, individual plateaus resembling the data from recorded neurons (Brierley et al., 1997a). An important characteristic of the model is that the frequency of bursting is directly affected by the amount of injected current (Fig. 3B, iii and iv). As we shall see later, this property allows synaptic input to the $\mathrm{N} 2 \mathrm{v}$ to regulate the timing of the plateau initiation and therefore the duration of the P phase of the feeding rhythm.

\section{N3t neurons are tonically active and show post-inhibitory rebound}

The N3t neurons fire during the third, swallow or S phase of the feeding cycle (Fig. 1). Part of this activity is due to the ability of the N3ts to show spontaneous tonic firing in the absence of synaptic input, but they possess additional post-inhibitory rebound (PIR) properties (Elliott \& Benjamin, 1985a). As shown in Fig. 3C (i), the N3t neuron receives inhibitory input during the $\mathrm{P}$ and $\mathrm{R}$ phases, which interrupts its normal tonic firing and entrains its activity to the feeding rhythm (Fig. 1). When the second, $\mathrm{R}$ phase of inhibition ceases, the N3t excitability is temporarily increased, leading to a short burst of spikes superimposed on a small $(5-10 \mathrm{mV})$ transient depolarization. Following this burst, the cell gradually returns to its normal mode of tonic activity.

In other neurons with PIR in the feeding network of Lymnaea (e.g. the B4 motoneurons), the presence of a hyperpolarization de-inactivated current, $i_{T}$, has been demonstrated (Straub \& Benjamin, 2001). $i_{T}$ is a low-threshold calcium current and it also underlies PIR phenomena in thalamocortical relay neurons (Smith et al., 2000). Based on this experimental evidence, we have assumed that the PIR properties of $\mathrm{N} 3 \mathrm{t}$ are also due to an $i_{T}$ current, located at the somatic compartment $\left(i_{X}=i_{T}\right)$. This current is dependent on two dynamic variables, one for the fast $(4 \mathrm{~ms})$ activation and one for the much slower $(400 \mathrm{~ms})$ inactivation of the current. In the absence of external stimuli, $i_{T}$ is mostly inactivated, but a small proportion 
remains active and provides a persistent depolarizing force that keeps the neuron in a state of tonic firing ( $\sim 4 \mathrm{~Hz}$, Fig. 3, Cii). If inhibitory stimuli of sufficient magnitude and duration are applied, part of the inactivation is removed. After the cell is released from inhibition, $i_{T}$ is strongly activated and it induces a transient increase in the firing frequency of the cell (Fig. 3, Cii). The gradual inactivation of $i_{T}$ at these depolarised membrane potentials allows the neuron to return to its basal tonic activity (Fig. 3, Cii). As shown in Fig. 3C, iii, the intensity of PIR is directly related to the strength of the applied hyperpolarizing current.

\section{SO modulatory interneuron}

SO is not necessary for the basic generation of the feeding pattern. However, when it is active, high-frequency, regular rhythms are invoked, similar to those seen in the intact animal in response to food (Elliott \& Benjamin, 1985b; Kemenes \& Elliott, 1994; Kemenes et al., 2001). Unlike the CPG interneurons, which occur as a bilaterally symmetrical pair, there is only one SO in each snail (either in the left or right buccal ganglion) and this single neuron can be used to initiate and maintain high frequency rhythms in isolated CNS preparations. In comparison to the previous neurons, $\mathrm{SO}$ is relatively simple to model as it fires in a tonic manner in response to artificial stimulation and has no further significant intrinsic properties (Straub et al., 2002; Fig. 3D, i). The somatic compartment of the model was assumed to be passive (it contains only a leakage current, thus $i_{X}=0$ ), and fires tonically under current clamp, at a frequency related to the magnitude of the injected current (Fig. 3D, ii and iii). In Fig. 3D (ii), the tonic firing of SO is interrupted repeatedly by brief inhibitory pulses, similar to those recorded during the natural feeding rhythm.

\section{Network properties and generation of the three phase feeding rhythm}

Recurrent inhibition between the N1M and N2v neurons is the source of rhythmicity in the feeding network

The CPG of Lymnaea was reconstructed from the modelled individual neurons on the basis of known synaptic connectivity obtained in previous electrophysiological experiments (Elliott \& Benjamin, 1985a). We began by connecting the N1M and N2v neurons, as it has been observed that co-cultured interneurons from the N1 and N2 groups form pairs with pattern generating abilities (Straub, 1999). In the intact nervous system, the N1M cells fire first followed by the N2v cells (Fig. 1). The N1M cells send excitatory synaptic input to the N2v neurons and N1M activity is then inhibited by feedback connections from the N2v cells (Elliott \& Benjamin, 1985a; Brierley et al., 1997b), i.e. N1M and N2v are connected via recurrent inhibition (Fig. 4A, i).

By implementing this connectivity pattern between the N1M and $\mathrm{N} 2 \mathrm{v}$ model neurons, we succeeded in modelling a two-component circuit capable of generating regular oscillations when appropriately stimulated. The sequence of events that leads to this repetitive pattern is summarized in Fig. 4A, ii. Constant current injection into N1M excites the neuron and eventually triggers a plateau potential. N1M activity slowly depolarizes $\mathrm{N} 2 \mathrm{v}$ due to the $\mathrm{N} 1 \mathrm{M} \rightarrow \mathrm{N} 2 \mathrm{v}$ cholinergic excitatory connection. This synapse was given a relatively large activation time constant ( $\tau=200 \mathrm{~ms}$ ) to mimic the synaptic connection observed in the real circuit (Elliott \& Benjamin, 1985a; Brierley et al., 1997b). In addition, we assumed a relatively low conductance value, which in combination with the property of the $\mathrm{N} 2 \mathrm{v}$ neurons that makes them oscillate at low frequencies (see Fig. 3B, iii), was sufficient to induce a slow depolarizing wave in $\mathrm{N} 2 \mathrm{v}$ (similar to that

\section{A The core $\mathrm{N} 1 \leftrightarrow \mathrm{N} 2$ pacemaker}

i) Network

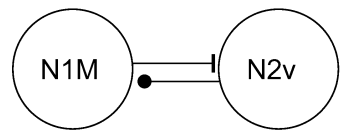

B The full triphasic feeding CPG

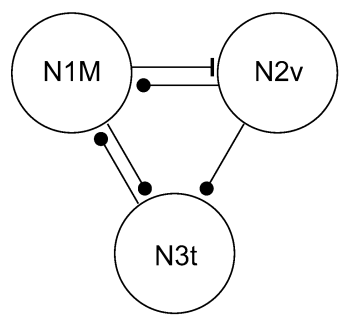

ii) Simulation

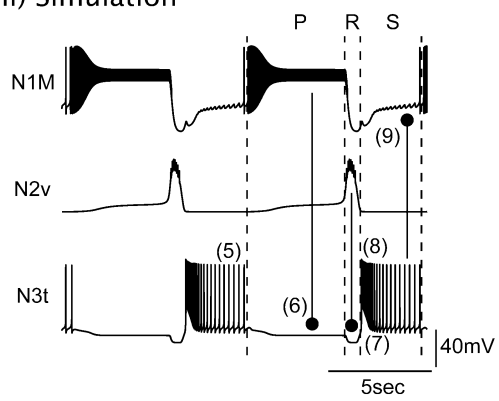

C The SO-driven oscillator i) Network

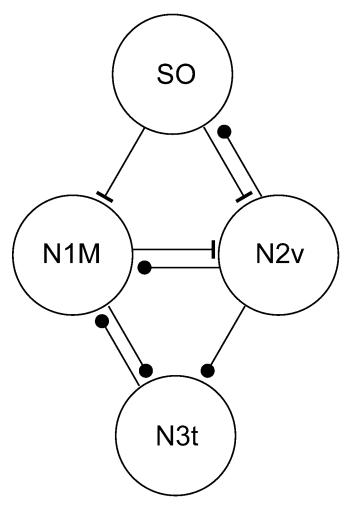

ii) Simulation

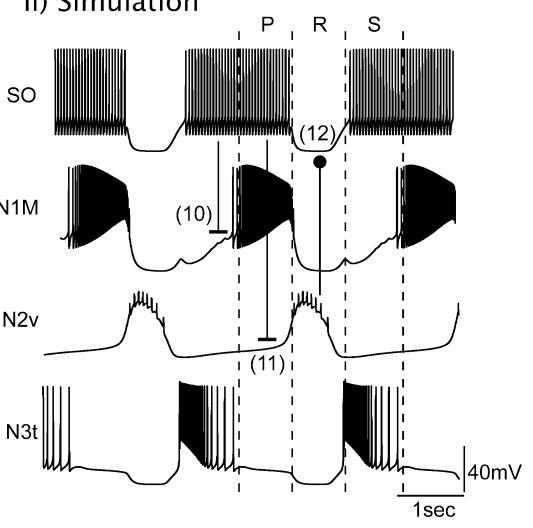

FIG. 4. Three levels of organization of the model network. (A) The twocomponent N1M/N2v pacemaker, (B) the full three-component $\mathrm{N} 1 \mathrm{M} / \mathrm{N} 2 \mathrm{v} / \mathrm{N} 3 \mathrm{t}$ feeding $\mathrm{CPG}$ and $(\mathrm{C})$ the SO-driven feeding CPG. The numbers in each of the right panels (ii) indicate the presence of important electrophysiological events during the generation of the feeding pattern. (1) Slow N1M-induced excitation and activation of the N2v; (2) recurrent inhibition of N1M during the R phase and rapid deactivation of its activity; (3) spontaneous termination of the N2v plateau ( $\sim 1 \mathrm{~s} \mathrm{long);} \mathrm{(4)} \mathrm{slow} \mathrm{recovery}$ of the N1M plateau, if external stimuli are still present; (5) spontaneous N3 tonic firing (in the absence of synaptic input); (6) suppression of the N3t activity, during the P phase; (7) further suppression of N3t during the R phase; (8) N3t generates a PIR response, when released from the R-phase inhibition; (9) delayed recovery of the N1M plateau during the S phase, due to N3timposed inhibitory input; (10) indirect, SO-mediated excitation of N1M; (11) SO contributes to the activation of the $\mathrm{N} 2 \mathrm{v}$ plateau, and (12) recurrent inhibition of SO, during the $\mathrm{R}$ phase.

observed in vitro, see Brierley et al., 1997b) and drive the neuron beyond the threshold for the generation of a plateau. The inhibitory effect of the N2-type neurons on N1M has been shown to be sufficiently strong to terminate the N1M plateau (Elliott \& Benjamin, 1985a). Thus, in the model, N2v activity hyperpolarizes N1M sufficiently for the de-activation of the current, which sustains the $\mathrm{N} 1 \mathrm{M}$ plateau $\left(i_{A C h}\right)$ and terminates its bursting. The $\mathrm{N} 2 \mathrm{v}$ plateau ceases spontaneously, approximately $1 \mathrm{~s}$ later and a new cycle of activity led by $\mathrm{N} 1 \mathrm{M}$ is initiated. 
Incorporation of the N3t gives rise to a three phase pattern of neuronal activity and shapes the swallow phase of the cycle

The feeding rhythm of Lymnaea is conventionally divided into three phases. During each phase only one type of CPG neuron (N1M, N2v or N3t) is active, while the remaining two are inhibited (Fig. 1). Consequently, a network capable of producing a triphasic rhythm must necessarily incorporate neurons of all three types. Thus, our next step was to introduce N3t into our artificial network.

In the intact nervous system, $\mathrm{N} 3 \mathrm{t}$ receives inhibitory synapses from $\mathrm{N} 1 \mathrm{M}$ and $\mathrm{N} 2 \mathrm{v}$ and, in turn, sends inhibitory input to N1M, but not to N2v, as shown in Fig. 4B, i (Elliott \& Benjamin, 1985a; Brierley et al., 1997b). The extended network that contains these additional elements is capable of producing a feeding rhythm consisting of three phases. The sequence of events that leads to the generation of a third phase of activity is summarized in Fig. 4B, ii. The activation of N1M by constant current injection results in the inhibition of $\mathrm{N} 3 \mathrm{t}$, thus interrupting its spontaneous tonic activity for the whole of the $\mathrm{P}$ phase of the rhythm. The induction of the $\mathrm{N} 2 \mathrm{v}$ plateau during the $\mathrm{R}$ phase imposes a second, stronger phase of inhibition upon N3t. The neuron remains inhibited until the spontaneous termination of the $\mathrm{N} 2 \mathrm{v}$ plateau. On the recovery from the R-phase inhibition, $i_{T}$ is significantly active, thus allowing N3t to fire a burst of spikes due to PIR, while gradually returning to its normal state of tonic activity. When N1M is finally re-activated, it inhibits N3t again and a new cycle begins. Briefly, the model predicts that the role of the P- and R-phase inhibitory input upon $\mathrm{N} 3 \mathrm{t}$ is to interrupt its normal tonic firing, leaving only a small window of phasic activity, which defines the S phase of the feeding rhythm.

\section{Driving of the three-cell rhythm by the SO}

Having constructed a basic network capable of producing triphasic rhythms, our next step was to extend it by adding the modulatory SO neuron to the model. SO sends excitatory input to N1M and N2v and receives inhibitory input from $\mathrm{N} 2 \mathrm{v}$, as shown in Fig. 4C, i (Elliott \& Benjamin, 1985b; Brierley et al., 1997b). Both the SO $\rightarrow$ N1M and $\mathrm{SO} \rightarrow \mathrm{N} 2 \mathrm{v}$ cholinergic excitatory synapses were given a relatively large activation time constant $(\tau=200 \mathrm{~ms})$ in order to simulate the slow depolarizing effects of SO upon the N1M and N2v interneurons (Elliott \& Benjamin, 1985b; Brierley et al., 1997b). Current injected into SO sustains a regular train of action potentials, which then drives $\mathrm{N} 1 \mathrm{M}$ towards the generation of a plateau (SO $\rightarrow$ N1M excitatory connection) and a cycle of spike activity. In parallel, $\mathrm{SO}$ contributes to the stimulation of $\mathrm{N} 2 \mathrm{v}$ ( $\mathrm{SO} \rightarrow \mathrm{N} 2 \mathrm{v}$ excitatory connection) during the whole duration of the $\mathrm{P}$ phase and accelerates its activation. With the transition to the $\mathrm{R}$ phase, $\mathrm{SO}$ is inhibited $(\mathrm{N} 2 \mathrm{v} \rightarrow \mathrm{SO}$ inhibitory connection), but it recovers after the spontaneous termination of the $\mathrm{N} 2 \mathrm{v}$ plateau and it starts to excite N1M during the swallow phase, eventually triggering a new cycle of network activity (Fig. 4C, ii).

\section{Manipulation of the CPG interneurons during fictive feeding phase-shifts the feeding rhythm}

Experiments in the intact nervous system have shown that brief current injections into feeding interneurons during the third (S) phase of SOdriven rhythms produce predictable phase shifts in the feeding pattern, providing further evidence that these cells are part of the CPG network (Elliott \& Benjamin, 1985a; Brierley et al., 1997b). In a similar manner, brief depolarising or hyperpolarising current pulses applied to interneurons of the model CPG during an SO-driven rhythm result in a characteristic phase shift and resetting of the feeding pattern (Fig. 5A). For example, the induction of an $\mathrm{N} 2 \mathrm{v}$ plateau in the interburst interval (S phase) by a depolarizing pulse delays the rhythm (Fig. 5A, i). A similar effect is observed when an N3t burst is induced, through application of a hyperpolarizing pulse at the same time point during the feeding cycle (Fig. 5A, ii). The model is therefore able to reproduce data from phase-resetting experiments, which is a further demonstration that it accurately captures the behaviour of the intact feeding CPG.

If prolonged hyperpolarization is applied to N1M sufficient to inhibit bursting activity for several cycles, then the rhythm in the rest of the network completely stops (Fig. 5B). This emphasizes that N1M is a key element of the feeding CPG and similar results were obtained in food driven rhythms by Kemenes et al. (2001).

\section{Dynamic network properties}

\section{Dual CPG and switching role for the N3t cells}

Staras et al. (2003) showed that N3t has an important role as a decision maker in determining the occurrence of feeding rhythms in the snail as well as being an important member of the CPG. In the absence of food, N3t fires tonically and suppresses rhythmic activity in the CPG by maintained inhibitory synaptic input to the N1M cells. Following presentation of food to the lips, the frequency of tonic inhibition upon N1M is gradually reduced (food hyperpolarizes N3t) and after a delay this reduction of inhibition, together with concomitant depolarization of N1M, was sufficient to change the network into a rhythmic mode, with $\mathrm{N} 3 \mathrm{t}$ switching function to fire phasically as part of the CPG.

This effect of food on N3t firing was mimicked in the model by injecting a ramp hyperpolarizing current into the N3t neuron. Similarly, the N1M was constantly injected with a small amount of steady depolarizing current to mimic the effect of applying food in the biological system (Kemenes et al., 2001). These manipulations induced a reduction in the tonic firing of N3t followed by a switch to rhythmic activity in both N3t and N1M (Fig. 6A), the reciprocal inhibitory connections between the two cells producing the expected alternating pattern of firing.

As well as switching the CPG pattern on or off, intermediate levels of CPG activity were seen in the absence of food, when the level of tonic activity in N3t was insufficient to completely inhibit the CPG. This occurred in preparations made from 'hungry' snails that are known to carry out spontaneous exploratory feeding movements (Staras et al., 2003). In the model, changing the tonic firing frequency of N3t from $3 \mathrm{~Hz}$ to more than $5 \mathrm{~Hz}$ induces a corresponding change in the frequency of the rhythm in the range $0-9$ cycles/min (Fig. 6B, i), which is in agreement with experimental data from semi-intact preparations (Fig. 6C from Staras et al., 2003). In this set of simulations, the PIR property of the model N3t was inactivated by setting $i_{T}=0$ (see Table 1). This was necessary, because PIR interferes with the tonic firing of N3t, when fictive feeding is fast, and makes it impossible to manipulate the frequency of tonic firing and correlate it to the frequency of the feeding rhythm. The absence of any significant PIR response was often the case in the biological neuron during similar manipulations [for example, see Fig. 1B (ii) and Fig. 4C from Staras et al., 2003]. By varying the N3t firing rate in the model, it was possible to reproduce the patterns of CPG activity monitored in the N1M corresponding in the natural system to satiety (no CPG activity, Fig. 6B, ii) and hungry animal with food present (highest-frequency CPG activity, Fig. 6B, iv). An intermediate band of $\mathrm{N} 3 \mathrm{t}$ frequencies $(\sim 5 \mathrm{~Hz})$ in the model corresponded to a hungry animal in the absence of food (Fig. 6B, iii), where spontaneous feeding movements take place at relatively large time intervals $(\sim 2-$ 3 cycles/min). This region is represented by the steep part of the 
A Phase-shift experiments

i) N2v-mediated phase-shift

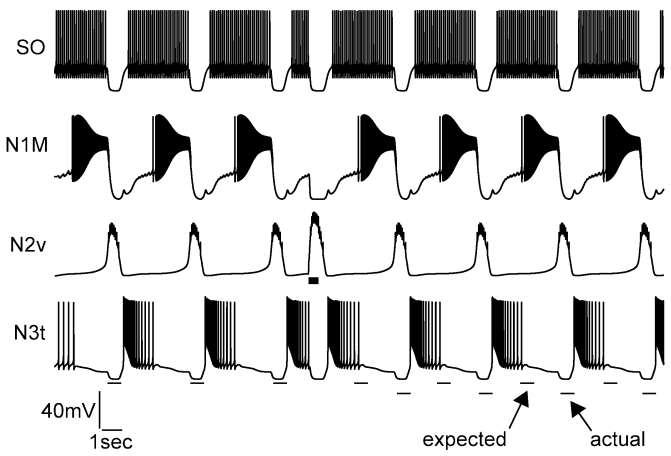

ii) N3t-mediated phase-shift

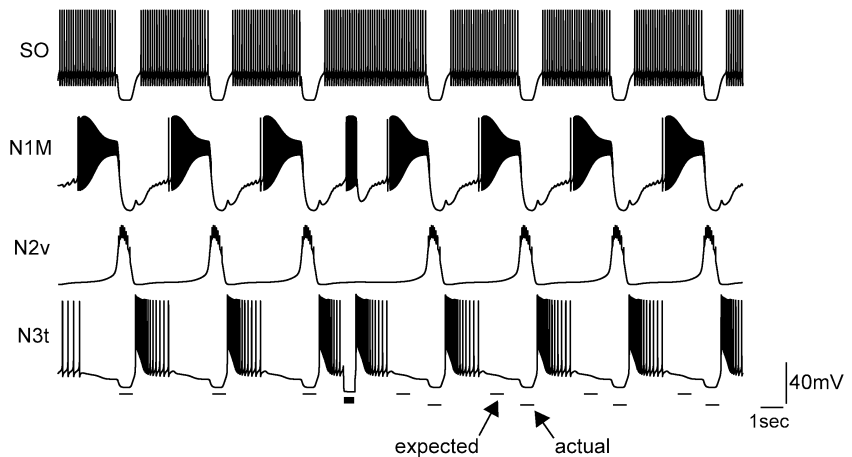

B Long-term suppression of the N1M burst

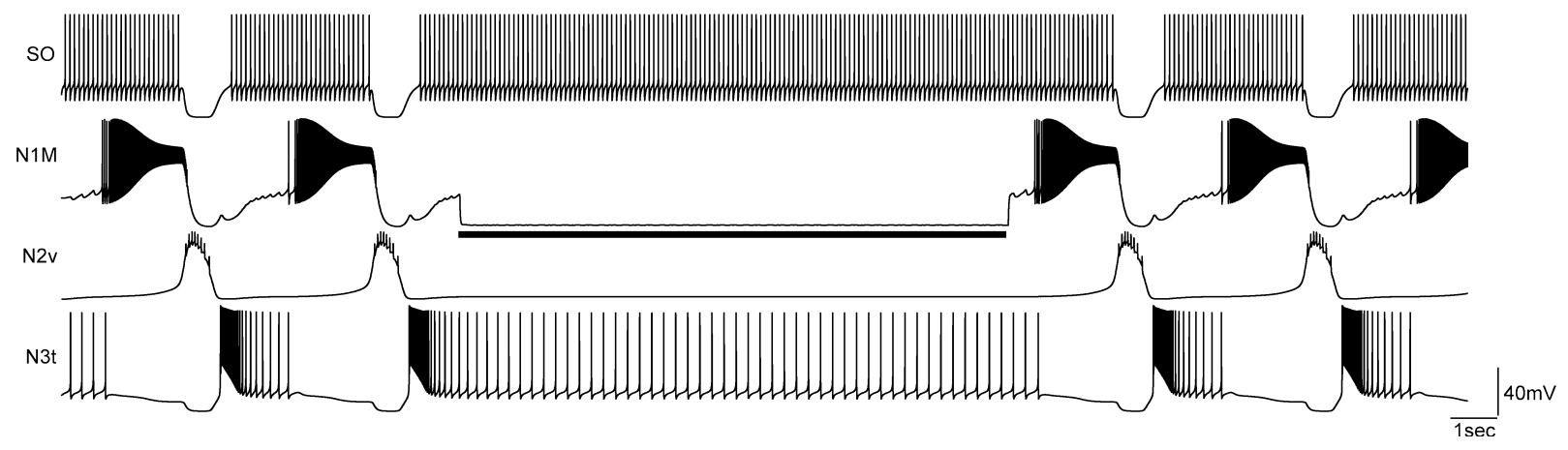

FIG. 5. (A) Application of brief depolarizing and hyperpolarizing current pulses to N2v and N3t interneurons in the model network during SO-driven fictive feeding resets the feeding rhythm. (Ai) A brief depolarizing current pulse applied to N2v during the interburst interval induces an early burst and delays the feeding rhythm. (Aii) Similarly, induction of an early N3t burst through the application of an inhibitory pulse delays the rhythm. (B) Maintained hyperpolarization of N1M stops the rhythm for as long as activity in N1M is suppressed.

curve in Fig. 6B, i marking the transition of the network between two different modes of operation (i.e. quiescence and fast, regular feeding activity).

In summary, the behaviour of the model is in agreement with previous experimental results, which suggest a dual role of $\mathrm{N} 3 \mathrm{t}$ as a pattern-generating/regulatory element of the feeding network in Lymnaea. The results from the modelling support the original hypothesis that it has a switching role in feeding. Importantly, the effect of behavioural state was also mimicked accurately by the model.

\section{The SO can maintain a high frequency feeding rhythm unlike the} NIM CPG

A striking characteristic of SO in the nervous system is its ability to drive fast regular rhythms with a lower cycle period (range $\sim 3-6 \mathrm{~s}$ ) compared to the N1M-driven ones (range $\sim 6-20 \mathrm{~s}$ ) (Elliott \& Benjamin, 1985b; Kemenes et al., 2001). A similar difference in the ability to drive feeding rhythms was found in the model feeding network. In the example shown in Fig. 7A, either NIM (Fig. 7A, i) or SO (Fig. 7A, ii) were stimulated with constant current injection to produce the maximum frequency of feeding cycles. Under these conditions, NIM achieved a minimum period of $\sim 6 \mathrm{~s}$, whereas the minimum period of the SO-driven feeding activity was $\sim 3 \mathrm{~s}$ (Fig. 7A, ii).

A further type of analysis involved comparing the duration of the three phases of the feeding rhythm ( $\mathrm{P}, \mathrm{R}$ and $\mathrm{S}$ phases shown in Fig. 7A, i) when driven by either N1M or SO as a function of cycle period. In the case of N1M-driven patterns, variations in period could be directly related to the duration of the $S$ phase (Fig. 7B, iii) with no changes in the $\mathrm{P}$ or $\mathrm{R}$ phases (Fig. $7 \mathrm{~B}$, $\mathrm{i}$ and ii). In contrast, in the SO-driven pattern (Fig. 7B, iv-vi), both the $\mathrm{P}$ and $\mathrm{S}$ phases were affected (Fig. 7B, iv and vi) with again no change in the $R$ phase (Fig. 7B, v). These results are in agreement with observations made by Elliott \& Andrew (1991) in semi-intact preparations, who found similar relations between the duration of the three phases of the rhythm and the cycle period during N1M and SO-driven patterns.

What is the basis of the differential ability between SO and N1M to drive the feeding pattern in our simulations? When current is directly injected into N1M, SO remains inactive (Fig. 7A, i) and the only source of excitation for $\mathrm{N} 2 \mathrm{v}$ is N1M itself. The weak N1M $\rightarrow \mathrm{N} 2 \mathrm{v}$ connection causes a slow depolarizing wave upon $\mathrm{N} 2 \mathrm{v}$, eventually triggering a plateau potential. The onset of the plateau coincides with the termination of the P phase of the feeding cycle. The relative insensitivity of the N1M plateau to injected current (see Predictions below), means that the effect of $\mathrm{N} 1 \mathrm{M}$ excitation upon $\mathrm{N} 2 \mathrm{v}$ and, in consequence, the length of the protraction phase is largely insensitive to external current application on $\mathrm{N} 1 \mathrm{M}$, even when the intensity of the stimulus is maximized. However, during an SO-driven rhythm, additional excitation is received by $\mathrm{N} 2 \mathrm{v}$, due to the direct $\mathrm{SO} \rightarrow \mathrm{N} 2 \mathrm{v}$ synapse, which accelerates the onset of the $\mathrm{N} 2 \mathrm{v}$ plateau and leads to the early termination of the N1M activity. For example, increasing the magnitude of the $\mathrm{SO} \rightarrow \mathrm{N} 2 \mathrm{v}$ conductance (range $0-1$ units, Fig. 7C) reduces the duration of the protraction phase when SO is maximally stimulated. This results in the generation of feeding cycles with correspondingly shorter period, when compared to the N1M-driven ones. 


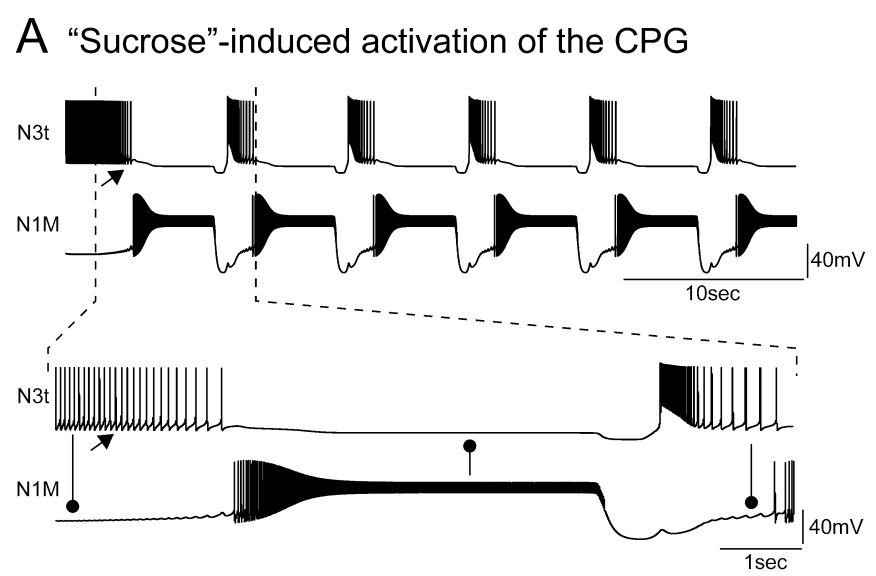

B Gating role of the N3t

i) Feeding freq. vs N3t firing freq.

ii) Satiated animal
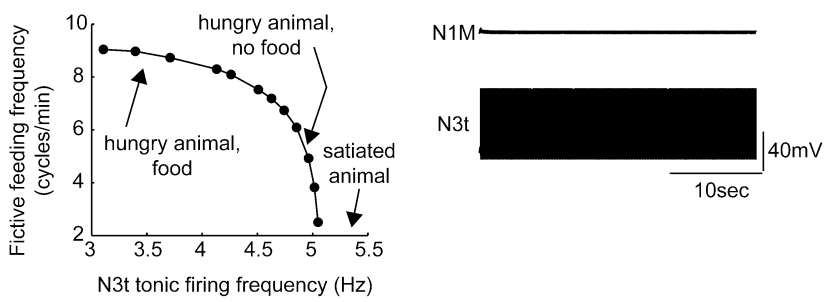

iii) Hungry animal, no food

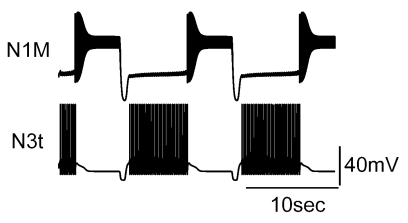

iv) Hungry animal, food

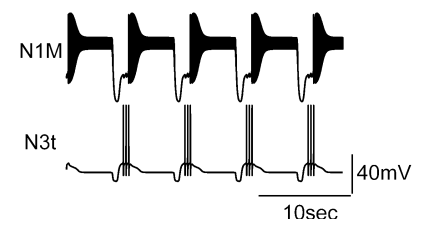

FIG. 6. Modelling the dual pattern-generating/switching role of N3t. (A) Simulation of the sucrose-induced activation of the CPG. In satiated animals, N3t keeps the feeding network under its suppressive control. Application of 'sucrose' (hyperpolarizing ramp current) to the system (arrows) reduces the frequency of the $\mathrm{N} 3$ tonic firing, which releases the CPG from inhibition and leaves N3t as part of the rhythmogenic circuit, firing in alternating bursts with N1M. Expanded pattern (below) shows the transition between suppression of the feeding pattern and the change to rhythmic firing. (B) Manipulating the tonic firing frequency of the model N3t controls the total frequency of the feeding pattern (Bi), with different levels of N3t activity corresponding to different physiological states of the animal (Bii-iv). Notice the absence of any significant post-inhibitory rebound response in this set of simulations (see main text for justification and Table 1).

\section{Predictions}

Further analysis of our model revealed new types of dynamic responses to neuronal stimulation, which were subsequently confirmed experimentally in the intact nervous system.

First, it was noted that injection of a constant depolarizing current into $\mathrm{N} 2 \mathrm{v}$ during slow N1M-driven feeding activity in our model reduced the overall cycle period (Fig. 8A, i). Therefore, it was predicted that constant current injection into the $\mathrm{N} 2 \mathrm{v}$ in the nervous system could also reduce the cycle period of the intact feeding CPG. The comparison of records from an $\mathrm{N} 2 \mathrm{v}$ neuron in the intact nervous system without and with current injection of $9 \mathrm{nA}$ showed indeed a reduction in the feeding cycle period (Fig. 8A, ii). At the population level, current injection significantly reduced the cycle period from $6.2 \pm 0.18 \mathrm{~s}$ to $4.1 \pm 0.2 \mathrm{~s}$ (paired $t$-test $P<0.001, n=5$ ).
Second, it was shown in the model that varying the level of current injection into $\mathrm{N} 1 \mathrm{M}$ had little effect on the firing rate of the $\mathrm{N} 1 \mathrm{M}$ cell and the duration of the protraction phase (Fig. 8B, i; see also Fig. 3A, iv). Comparisons were made between $3 \mathrm{nA}$ and $6 \mathrm{nA}$ levels of current injection superimposed on a minimum bias depolarizing current that was used to maintain a slow feeding rhythm ( $\sim 19 \mathrm{~s})$. The N1M burst produced by the biasing current alone was labelled 'spontaneous' in Fig. $8 \mathrm{~B}$, i, because it produced low frequency $\mathrm{N} 1 \mathrm{M}$ activity that mimics the spontaneous activity often recorded in the natural system. Although a small decrease $(\sim 500 \mathrm{~ms})$ in the duration of the protraction phase was observed (due to the compression of the initial transitory phase of the plateau at higher current injection levels), it was not significant when compared to the overall change in the duration of the feeding rhythm ( $\sim 13 \mathrm{~s}$ ), which was mainly due to changes in the duration of the third (swallow) phase (see also Fig. 7). When the same levels of depolarizing current were applied to N1M during a spontaneous biological rhythm, a similar result was obtained (Fig. 8B, ii). A oneway ANOVA showed that there was no significant difference in the mean levels of firing in the neuron when the results from five cells were compared $\left(F_{2,12}=0.41, P=0.68\right)$. Neither was there any significant difference in the duration of the N1M burst (measure of the protraction phase of the feeding rhythm) in the neuron $\left(F_{2,12}=2.33, P=0.15\right)$, as predicted by the model.

\section{Discussion}

The feeding network of Lymnaea stagnalis has been the subject of extensive electrophysiological studies, which have revealed a rich set of intrinsic properties and synaptic connections between the component neurons. The feeding CPG is dynamically regulated by at least two types of neurons. One is involved in the switching of the feeding network from quiescence to rhythmic activity and the other is important in controlling the frequency of the feeding oscillator. By wiring into the model the known synaptic connectivity of these two types of regulatory interneurons, we were able to recapitulate quantitatively the biological functions of these two cells and verify the usefulness of the basic CPG model for studying the network dynamics.

\section{Modelling the three phase feeding rhythm}

At the network level, the core of the Lymnaea model system is a twocomponent circuit composed of the N1M and N2v interneurons, connected via recurrent inhibition. The crucial features for the function of this two-cell pacemaker are the very slow induction of the $\mathrm{N} 2 \mathrm{v}$ plateau by N1M and the termination of the N1M plateau by N2v. The former is possible due to the weak and slow N1M $\rightarrow \mathrm{N} 2 \mathrm{v}$ excitatory synapse and the ability of the $\mathrm{N} 2 \mathrm{v}$ to oscillate at arbitrarily low frequencies. The latter is possible due to the strong $\mathrm{N} 2 \mathrm{v} \rightarrow \mathrm{N} 1 \mathrm{M}$ inhibition, which drives the $\mathrm{N} 1 \mathrm{M}$ membrane at very negative potentials and rapidly deactivates the N1M plateau. The inability to significantly affect the firing frequency of N1M by varying the level of injected current means that the duration of the $\mathrm{P}$ phase of the rhythm will be largely unchanged. Thus any modifications in the duration of the whole feeding cycle arise from regulation of the time period it takes the N1M plateau to recover from the N2v inhibition. Addition of the N3t neuron with its reciprocal inhibitory synaptic connectivity to the other CPG neurons results in the successful production of the three-phase CPG rhythm, which is characteristic of the biological feeding network. 


\section{A Maximal stimulation of the network}

i) N1M-driven rhythm

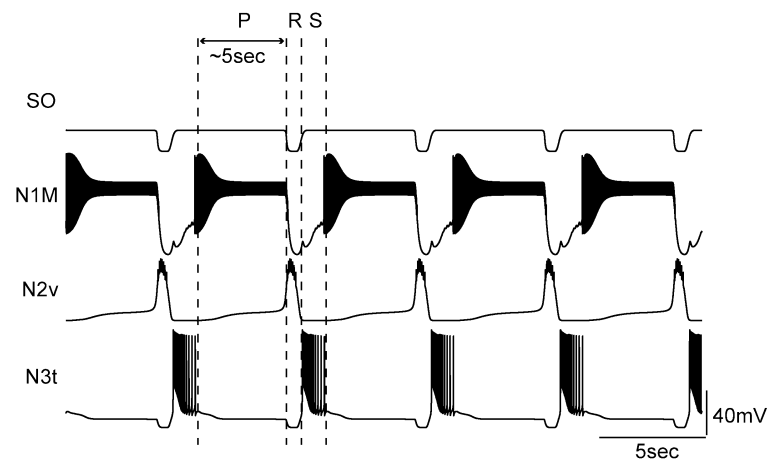

ii) SO-driven rhythm

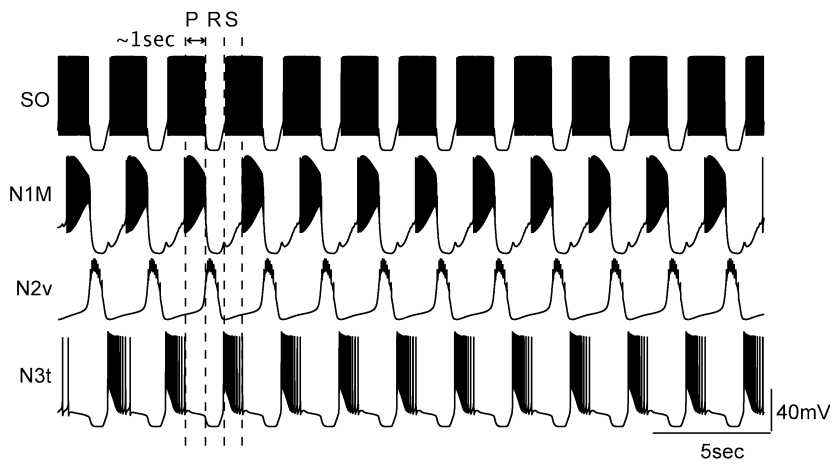

B Phase duration vs cycle period

i) Protraction

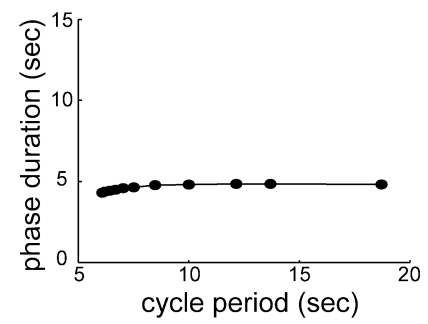

iv) Protraction

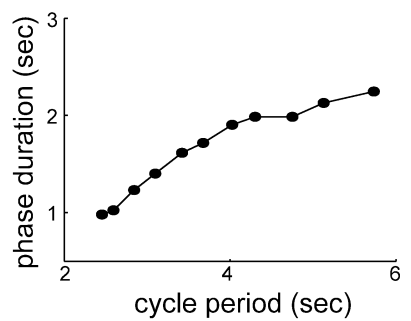

ii) Rasp

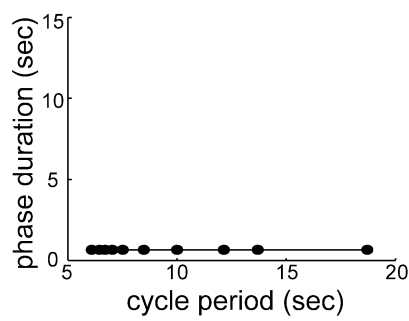

v) Rasp

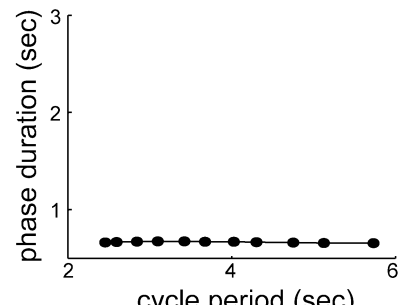

cycle period (sec) iii) Swallow

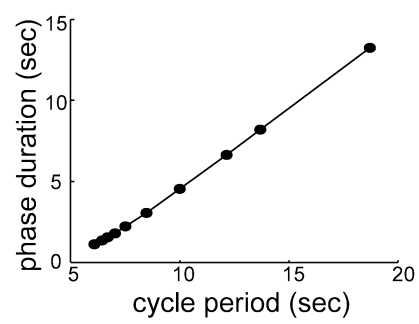

vi) Swallow

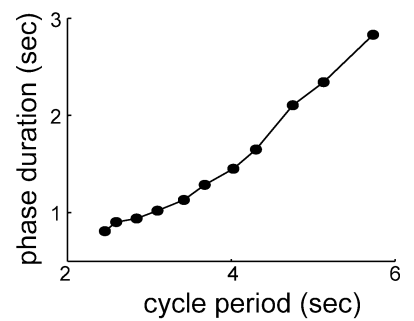

N1M-driven

SO-driven

\section{The effect of the $\mathrm{SO} \rightarrow \mathrm{N} 2 \mathrm{v}$ conductance}

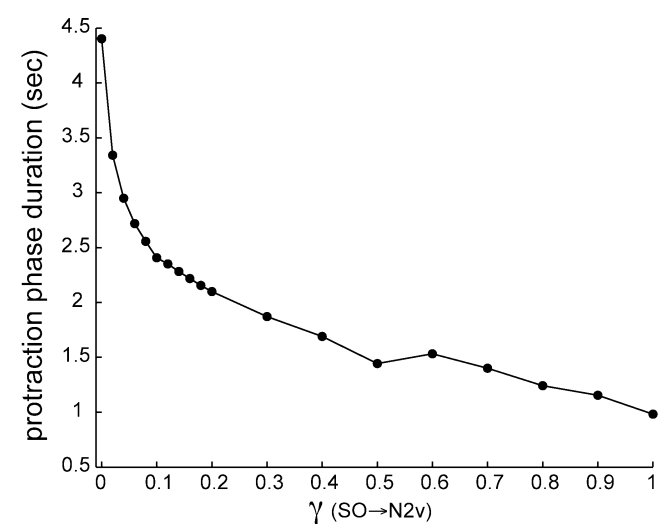

FIG. 7. Activation of the feeding CPG by current injection into SO induces higher-frequency rhythms than N1M by reducing the duration of the protraction phase. (A) Injecting continuous depolarizing current into SO drives a higher frequency rhythm (Aii) than N1M (Ai) when the network is maximally stimulated to achieve its highest frequency of oscillation. The duration of the rasp and swallow phase remains approximately the same with either cell stimulated ( $\sim 1 \mathrm{~s}$ each). However, the duration of the protraction phase is much shorter when $\mathrm{SO}$ is generating the rhythm $(\sim 1 \mathrm{~s})$ compared to N1M $(\sim 5 \mathrm{~s})$, accounting for the higher frequency rhythm. (B) The duration of the three phases of the feeding cycle as a function of the overall duration (period) of the rhythm, during N1M (Bi-iii) and SO-driven patterns (Biv-vi). The period of the rhythm was controlled by maintained current injections of different amplitudes in either N1M or SO. In the N1M-driven patterns, changes in the duration of the cycle are solely due to changes in the duration of the swallow phase. The period of the rhythm does not fall below $\sim 6 \mathrm{~s}$. However, during SO-driven patterns, changes in the duration of the protraction phase contribute significantly to changes in the total duration of the cycle, which reaches a minimum value of $\sim 3 \mathrm{~s}$. (C) Dependence of the protraction phase duration on the magnitude of the SO $\rightarrow$ N2v excitatory conductance. Larger values of this parameter allow for a protraction phase of shorter duration, when SO in maximally stimulated. 


\section{A Response of the network to N2v stimulation}

i) Model

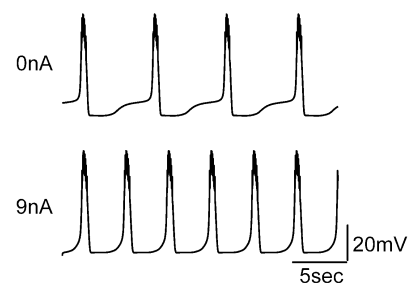

ii) Recording

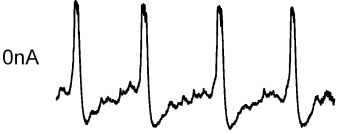

$9 n A$

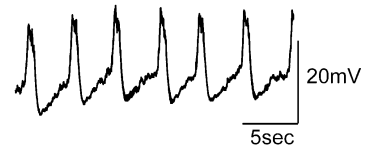

B Response of the N1M neuron to injected current

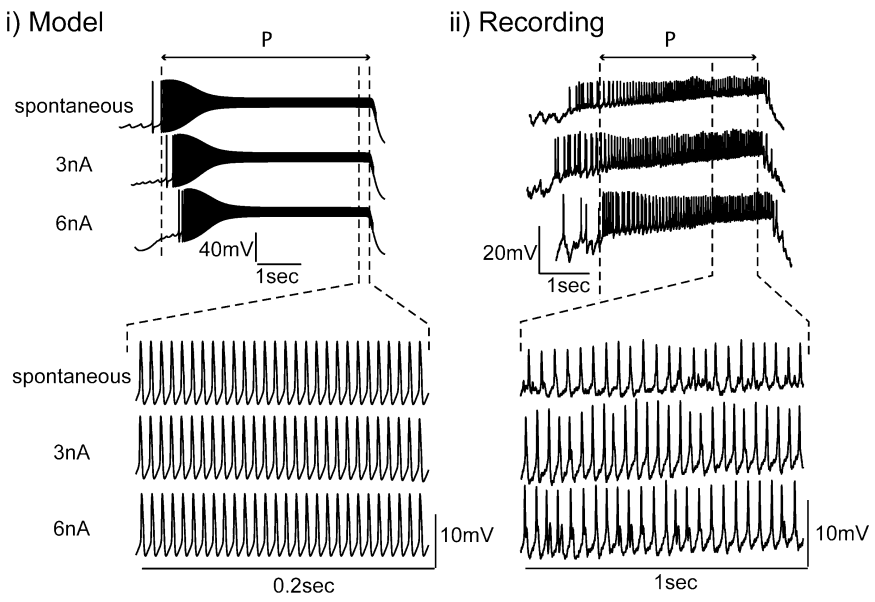

FIG. 8. Predictions from the model are tested by electrophysiological experiments. (A) Current injection into $\mathrm{N} 2 \mathrm{v}$ increases the frequency of the feeding rhythm. The model predicts (Ai) that stimulating N2v will transform a slow rhythm (period $\sim 6.5 \mathrm{~s}$, top) to a fast one (period $\sim 4 \mathrm{~s}$, bottom). This prediction is confirmed from experiments in the natural network (Aii, top, no current injection; bottom, current injection). (B) N1M activity is relatively constant in response to various levels of injected current. Stimulating the model neuron $(\mathrm{Bi})$ with two different levels of depolarizing current does not significantly affect its maximal firing frequency or the duration of its plateau activity (indicative of the duration of the $\mathrm{P}$ phase). In the biological network, similar manipulation of N1M during a spontaneous rhythm (Bii) reveals a similar insensitivity of the neuron to injected current. The spontaneous activity of the natural system was mimicked in the model by maintaining a slow pattern $(\sim 19 \mathrm{~s})$ via injection of a minimal bias current into N1M. This current corresponds to the background excitation related to behavioural state that is required for the induction of spontaneous activity in the natural system.

\section{Dynamic regulation of the feeding network}

Staras et al. (2003) proposed that the regulation of N3t tonic firing plays a central role in the occurrence of episodes of activity in an otherwise quiescent CPG. In particular, they found that when N3t fires tonically, it suppresses the rest of the network through its inhibitory effect upon N1M. When the firing frequency of N3t is reduced (for example, when food is presented to the system and/or the levels of hunger are increased), the network starts oscillating. When the N3t is artificially suppressed, the CPG is released from inhibition and it enters a period of normal rhythmic activity. These results are in agreement with various features of the model presented here. By manipulating the firing levels of $\mathrm{N} 3 \mathrm{t}$ we were able to mimic the suppressive effect of $\mathrm{N} 3 \mathrm{t}$ activity at the appropriate 'physiological' level of tonic firing and then by reducing the level of tonic firing to switch the network to rhythmic activity with $\mathrm{N} 3 \mathrm{t}$ firing in a burst pattern as part of the CPG. Staras et al. (2003) also showed that firing rates in the N3t cell (and therefore its ability to inhibit the feeding rhythm) was related to the satiety levels of the animal (hungry animals demonstrate lower tonic firing frequency) and again this could be mimicked in the model by setting the level of $\mathrm{N} 3 \mathrm{t}$ firing at varying levels of activity within the physiological range. The Lymnaea model offers a simple but effective mechanism for switching between activity and inactivity, with the same N3t neuron utilized for both rhythm generation and suppression depending on the level of tonic firing in the cell.

The other aspect of dynamic network function that was successfully modelled was the frequency control mechanism of SO. The previous quantitative analysis of frequency control by Elliott \& Andrew (1991) compared the duration of each of the three phases of the feeding rhythm (P, R and S) with different cycle periods. During N1M-driven rhythms when SO was silent, the duration of the $\mathrm{S}$ phase varied consistently with the cycle period, whilst the duration of the $\mathrm{P}$ and $\mathrm{R}$ phases did not change. However, in the case of the faster SO-driven rhythms, both the $\mathrm{P}$ and $\mathrm{S}$ phases varied significantly with the duration of the whole cycle. The $\mathrm{R}$ phase again remained unchanged. These observations are in good agreement with the results of our model. It is interesting that an analysis of the effects of the cerebral giant cells (another type of modulatory interneuron) on high-frequency SOdriven rhythms also showed significant reductions in the duration of the P phase (Yeoman et al., 1996).

\section{Comparison with other systems}

Other models for behavioural switching of episodic rhythmic behaviour depend on the concept of positive activation of the rhythm. The previously quiescent animal (or preparation) is activated by sensory input to extrinsic command neurons (e.g. Frost \& Katz, 1996) or centres (e.g. Grillner, 2003) that then excite the CPG network. Quiescence in these systems is a 'default' inactive state occurring in the absence of excitatory input. What is suggested by the Lymnaea model is that quiescence is an active process where tonic inhibition suppresses the feeding CPG. A similar type of active inhibition has recently been described on the hatchling frog tadpole where rhythmic swimming is suppressed by descending reticulo-spinal GABAergic neurons that inhibit rhythmic activity in the spinal CPG circuitry (Lambert et al., 2004). This 'stopping' of swimming behaviour is due to sensory excitatory input to the GABAergic neurons from mechanosensory neurons located on the head surface of the tadpole. The head of the tadpole remains attached by a cement gland to a substrate during quiescence and this provides tonic inhibition to the swim circuit, thus preventing swimming behaviour. In Lymnaea, tonic inhibition arises from a CPG interneuron that fires continuously in the absence of food input. It is believed that this tonic activity is intrinsically produced although there must be some modulation of the level of N3t tonic activity by sensory input (from internal sensors) because the frequency of N3t firing is modified by 'internal state' (hunger and satiety, Staras et al., 2003). In the general history of behavioural neurobiology the concept of active inhibition leading to reduced responsiveness is a well-developed concept (e.g. Krasne \& Wine, 1975) but only rarely has the neural mechanism been investigated and we know of no example where the cellular mechanism of inhibition mechanism has been supported by modelling studies.

Other examples of behavioural switching depend on more complex features of network function (reviewed in Calabrese, 2003) because more than one behaviour is involved. A good example comes from the mollusc Aplysia, whose feeding CPG is capable of producing at least two different types of motor programs, ingestion and egestion, which involve protraction-retraction and opening-closing movements of the radula. A comprehensive neural model of motor program switching between these two different modes of operation (Jing \& Weiss, 2001) depends on the interplay between three neurons, the non-CPG CBI-3 
and the $\mathrm{B} 20$ and $\mathrm{B} 4 / 5 \mathrm{CPG}$ elements, which are embedded in a circuit of a total of nine neurons. It is believed that the switching between ingestion and egestion is induced through the CBI-3. This interneuron inhibits the B20 and B4/5 units, which have to be active for egestion to take place. Thus, manipulation of a single neuron controls the state of activity of two critical elements of the feeding network (i.e. B20 and B4/5) and promotes the switching between two different modes of operation.

Other examples of frequency-control mechanisms come from systems that employ reciprocal inhibition between intrinsic oscillators as a central design feature. Frequency control often involves the regulation of the intrinsic properties of the generating cells determining the duration of the alternating bursts (Selverston et al., 1997; Marder, 2000). For example, in the swim CPG of Clione, serotonin induces a spike-narrowing effect, which reduces the time interval that each of the mutually inhibited oscillatory neurons is active in and, thus, the frequency of alternating bursts (Satterlie et al., 2000). In the case of the crustacean stomatogastric ganglion, various neuromodulators, such as dopamine, octopamine, serotonin or the neuropeptide proctolin, are believed to modify various intrinsic and synaptic properties of the pacemaker neurons that form the pyloric circuit (for example, the intrinsic oscillators frequency, the characteristics of the post-inhibitory rebound property and the resting membrane potential of the component cells), leading to corresponding changes in the frequency of the pyloric rhythm (Hooper \& Marder, 1987; Kepler et al., 1990; Ayali \& HarrisWarrick, 1999; Makino et al., 2000). Synaptic interactions are also important in determining the frequency of rhythmically active networks, for example in the pyloric network of the lobster. In an important modelling study, Nadim et al. (1999) have shown that the inclusion of synaptic depression in a network of two mutually inhibited neurons, one of which is a pacemaker, leads to a configuration which conditionally demonstrates oscillations at a frequency largely determined by specific synaptic characteristics of the model network.

In conclusion, we have constructed a minimal model of the Lymnaea feeding CPG circuit, which mimics important aspects of the behaviour of the biological system, both at the single neuron and network levels. Future developments may include scaling up the model through the addition of more identified pattern-generating interneurons, motorneurons and modulatory interneurons and the implementation of the bilateral symmetry that characterizes much of the biological circuit. This extended model would be useful as a platform for studying the issues of robustness and dynamic control in the network, with potentially interesting engineering applications. Also, analysis of the model could provide insight into the action of various neuromodulators found in the biological system, such as acetylcholine and serotonin, and in locating possible 'sites' within the network (synapes and intrinsic neuronal properties), which could undergo short- or long-term plastic changes as a result of experience (Kemenes et al., 2006).

\section{Acknowledgements}

This research was supported by the Engineering and Physical Sciences Research Council (EPSRC) and the Biotechnology and Biological Sciences Research Council (BBSRC), United Kingdom. GK was also supported by the Medical Research Council (MRC), United Kingdom.

\section{Abbreviations}

$\mathrm{ACh}$, acetylcholine; CPG, central pattern generator; N1M, N1 medial; N2v, N2 ventral; N3t, N3 tonic; PIR, post-inhibitory rebound; P, protraction; R, rasp; S, swallow; SO, slow oscillator.

\section{References}

Abbott, L. \& Marder, E. (1998) Modelling small networks. In Koch, C. \& Segev, I., (Eds), Methods in Neuronal Modelling: from Ions to Networks. MIT Press, Cambridge MA, pp 361-410.

Ayali, A. \& Harris-Warrick, R.M. (1999) Monoamine control of the pacemaker kernel and cycle frequency in the lobster pyloric network. J. Neurosci., 19, 6712-6722.

Benjamin, P.R. \& Rose, R.M. (1979) Central generation of bursting in the feeding system of the snail, Lymnaea stagnalis. J. Exp. Biol., 80, 93-118.

Booth, V., Rinzel, J. \& Kiehn, O. (1997) Compartmental model of vertebrate motorneurons for $\mathrm{Ca}^{2+}$-dependent spiking and plateau potentials under pharmacological treatment. J. Neurophysiol., 78, 3371-3385.

Brierley, M.J., Staras, K. \& Benjamin, P.R. (1997a) Behavioural function of glutamatergic interneurons in the feeding system of Lymnaea: plateauing properties and synaptic connections with the motor neurons. J. Neurophysiol., 78, 3386-3395.

Brierley, M.J., Yeoman, M.S. \& Benjamin, P.R. (1997b) Glutamatergic N2v cells are central pattern generator interneurons of the Lymnaea feeding system: new model for rhythm generation. J. Neurophysiol., 78, 3396-3407.

Calabrese, R.L. (2003) Behavioural choices: how neuronal networks make decisions. Curr. Biol., 13, R140-R142.

Destexhe, A. \& Marder, E. (2004) Plasticity in single neuron and circuit computations. Nature, 431, 789-795.

Elliott, C.J.H. \& Andrew, T. (1991) Temporal analysis of snail feeding rhythms: a three-phase relaxation oscillator. J. Exp. Biol., 157, 391-408.

Elliott, C.J.H. \& Benjamin, P.R. (1985a) Interactions of pattern-generating interneurons controlling feeding in Lymnaea stagnalis. J. Neurophysiol., 54, 1396-1411.

Elliott, C.J.H. \& Benjamin, P.R. (1985b) Interactions of the slow oscillator interneuron with feeding pattern-generating interneurons in Lymnaea stagnalis. J. Neurophysiol., 54, 1412-1421.

Frost, W.N. \& Katz, P.S. (1996) Single neuron control over a complex motor program. Proc. Natl Acad. Sci. USA, 93, 422-426.

Galassi, M., Davies, J., Theiler, J., Gough, B., Jungman, G., Booth, M. \& Rossi, F. (2005) GNU Scientific Library Reference Manual, 2nd Edn. Network Theory Ltd, UK.

Golowasch, J., Casey, M., Abbott, L.F. \& Marder, E. (1999) Network stability from activity-dependent regulation of neuronal conductances. Neural Comput., 11, 1079-1096.

Grillner, S. (2003) The motor infrastructure: from ion channels to neuronal networks. Nature Rev. Neurosci., 4, 573-586.

Hodgkin, A.L. (1948) The local electric changes associated with repetitive action in a non-medulated axon. J. Physiol., 107, 165-181.

Hodgkin, A.L. \& Huxley, A.F. (1952) A quantitative description of membrane current and application to conduction and excitation in nerve. J. Physiol., 117, 500-544.

Hooper, S.L. \& Marder, E. (1987) Modulation of the lobster pyloric rhythm by the peptide proctolin. J. Neurosci., 7, 2097-2112.

Jing, J. \& Weiss, K.R. (2001) Neural mechanisms of motor program switching in Aplysia. J. Neurosci., 21, 7349-7362.

Katz, P.S. \& Frost, W.N. (1996) Intrinsic neuromodulation: altering neuronal circuits from within. TINS, 19, 54-61.

Kemenes, G. \& Elliott, C.J.H. (1994) Analysis of the feeding motor pattern in the pond snail, Lymnaea stagnalis: Photoinactivation of axonally stained pattern-generating interneurons. J. Neurosci., 14, 153-166.

Kemenes, G., Staras, K. \& Benjamin, P.R. (2001) Multiple types of control by identified interneurons in a sensory-activated rhythmic motor pattern. J. Neurosci., 21, 2903-2911.

Kemenes, I., Straub, V.A., Nikitin, E.S., Staras, K., O’Shea, M., Kemenes, G. \& Benjamin, P.R. (2006) Role of delayed non-synaptic neuronal plasticity in long-term associative memory. Curr. Biol., 16, 1269-1279.

Kepler, T.B., Marder, E. \& Abbott, L.F. (1990) The effect of electrical coupling on the frequency of model neuronal oscillators. Science, 248, 83-85.

Krasne, F.B. \& Wine, J.J. (1975) Extrinsic modulation of crayfish escape behaviour. J. Exp. Biol., 63, 433-450.

Lambert, T.D., Li, W.C., Soffe, S.R. \& Roberts, A. (2004) Brainstem control of activity and responsiveness in resting frog tadpoles: tonic inhibition. J. Comp. Physiol. A, 190, 331-342.

Makino, Y., Akiyama, M. \& Yano, M. (2000) Emergent mechanisms in multiple pattern generation of the lobster pyloric network. Biol. Cybern., 82, 443-454.

Marder, E. (2000) Motor pattern generation. Curr. Opin. Neurobiol., 10, 691-698.

Marder, E. \& Abbott, L.F. (1995) Theory in motion. Curr. Opin. Neurobiol., 5, $832-840$. 
Marder, E. \& Calabrese, R.L. (1996) Principles of rhythmic motor pattern generation. Physiol. Rev, 76, 687-717.

Marder, E. \& Selverston, A.I. (1992) Modelling the stomatogastric nervous system. In Harris-Warrick, R.M., Marder, E., Selverston, A.I. \& Moulins, M., (Eds), Dynamic Biological Networks: the Stomatogastric Nervous System. MIT Press, Cambridge MA, pp. 161-196.

Nadim, F., Manor, Y., Kopell, N. \& Marder, E. (1999) Synaptic depression creates a switch that controls the frequency of an oscillatory circuit. Proc. Natl Acad. Sci. USA, 96, 8206-8211.

Rose, R.M. \& Benjamin, P.R. (1981) Interneuronal control of feeding in Lymnaea stagnalis. I. Initiation of feeding by a single buccal interneuron. J. Exp. Biol., 92, 187-201.

Satterlie, R.A., Norekian, T.P. \& Pirtle, T.J. (2000) Serotonin-induced spike narrowing in a locomotor pattern generator permits increases in cycle frequency during accelerations. J. Neurophysiol., 83, 2163-2170.

Selverston, A.I., Panchin, Y.V., Arshavsky, Y.I. \& Orlovsky, G.N. (1997) Shared features of invertebrate central pattern generators. In Stein, P.S.G., Grillner, S., Selverston, A.I. \& Stuart, D.G., (Eds), Neurons, Networks and Motor Behaviour. MIT Press, Cambridge MA, pp 105-117.

Smith, G.D., Cox, C.L., Sherman, S.M. \& Rinzel, J. (2000) Fourier analysis of sinusoidally driven thalamocortical relay neurons and a minimal integrateand-fire-or-burst model. J. Neurophysiol., 83, 588-610.

Soto-Trevino, C., Rabbah, P., Marder, E. \& Nadim, F. (2005) Computational model of electrically coupled, intrinsically distinct pacemaker neurons. J. Neurophysiol., 94, 590-604.

Staras, K., Gyori, J. \& Kemenes, G. (2002) Voltage-gated ionic currents in an identified modulatory cell type controlling molluscan feeding. Eur. $J$. Neurosci., 15, 109-119.
Staras, K., Kemenes, G. \& Benjamin, P.R. (1998) Pattern-generating role for motorneurons in a rhythmically active neuronal network. J. Neurosci., 18, 3669-3688.

Staras, K., Kemenes, I., Benjamin, P.R. \& Kemenes, G. (2003) Loss of selfinhibition is a cellular mechanism for episodic rhythmic behaviour. Curr Biol., 13, 116-124.

Straub, V.A. (1999) In vitro study of a central pattern generator. PhD Thesis. University of Sussex, UK.

Straub, V.A., Staras, K., Kemenes, G. \& Benjamin, P.R. (2002) Endogenous and network properties of Lymnaea feeding central pattern generator interneurons. J. Neurophysiol., 88, 1569-1583.

Straub. V.A. \& Benjamin, P.R. (2001) Extrinsic modulation and motor pattern generation in a feeding network: a cellular study. J. Neurosci., 21, 1767-1778.

Susswein, A.J., Hurwitz, I., Thorne, R., Byrne, J.H. \& Baxter, D.A. (2002) Mechanisms underlying fictive feeding in Aplysia: coupling between a large neuron with plateau potentials activity and a spiking neuron. J. Neurophysiol., 87, 2307-2323.

Wang, X.J. \& Buzsaki, G. (1996) Gamma oscillation by synaptic inhibition in a hippocampal interneuronal network model. J. Neurosci., 16, 6402-6413.

Wilson, H.R. (1999) Simplified dynamics of human and mammalian neocortical neurons. J. Theor. Biol., 200, 375-388.

Yeoman, M.S., Brierley, M.J. \& Benjamin, P.R. (1996) Central pattern generator interneurons are targets for the modulatory serotonergic cerebral giant cells in the feeding system of Lymnaea. J. Neurophysiol., 75, 11-25.

Yeoman, M.S., Vehovszky, G., Kemenes, G., Elliott, C.J. \& Benjamin, P.R (1995) Novel interneuron having hybrid modulatory-central pattern generator properties in the feeding systems of the snail, Lymnaea stagnalis. J. Neurophysiol., 73, 112-124. 